\title{
MicroRNA-26a-3p rescues depression-like behaviors in male rats via preventing hippocampal neuronal anomalies
}

\author{
Ye Li, ${ }^{1}$ Cuiqin Fan, ${ }^{1}$ Liyan Wang, ${ }^{2}$ Tian Lan, ${ }^{1}$ Rui Gao, ${ }^{3}$ Wenjing Wang, ${ }^{1}$ and Shu Yan Yu ${ }^{1,4}$ \\ 'Department of Physiology and 2Morphological Experimental Center, School of Basic Medical Sciences, Cheeloo College of Medicine, Shandong University, Jinan, Shandong, China. ${ }^{3}$ Department of \\ Microorganism, Jinan Nursing Vocational College, Lvyoulu Road, Jinan, Shandong Province, China. ${ }^{4}$ Shandong Key Laboratory of Mental Disorders, School of Basic Medical Sciences, Cheeloo College of \\ Medicine, Shandong University, Jinan, Shandong, China.
}

\begin{abstract}
Depression is a neuropsychiatric disease associated with neuronal anomalies within specific brain regions. In the present study, we screened microRNA (miRNA) expression profiles in the dentate gyrus (DG) of the hippocampus and found that miR26a-3p was markedly downregulated in a rat model of depression, whereas upregulation of miR-26a-3p within DG regions rescued the neuronal deterioration and depression-like phenotypes resulting from stress exposure, effects that appear to be mediated by the PTEN pathway. The knockdown of miR-26a-3p in DG regions of normal control rats induced depression-like behaviors, effects that were accompanied by activation of the PTEN/PI3K/Akt signaling pathway and neuronal deterioration via suppression of autophagy, impairments in synaptic plasticity, and promotion of neuronal apoptosis. In conclusion, these results suggest that miR-26a-3p deficits within the hippocampal DC mediated the neuronal anomalies contributing to the display of depression-like behaviors. This miRNA may serve as a potential therapeutic target for the treatment of depression.
\end{abstract}

\section{Introduction}

Depression is a prevalent psychiatric disorder related to structural and functional neuronal changes within specific brain regions (13). Currently, most clinical treatments for depression have focused on restoring dysregulated monoamine neurotransmitter systems within the brain $(4,5)$. However, the limited benefits associated with such treatments indicate that more complicated mechanisms are involved in the etiology of depression (6-8). Previous evidence from clinical studies has indicated that disruptions in the normal structural and functional homeostasis in specific brain regions may be involved in the progression of depression in patients $(1,9)$. Consistently, accumulating results from animal models of depression have indicated that stressful stimuli produce neuronal injury, for example increased apoptosis within the hippocampus or medial prefrontal cortex, and thus result in the display of depression-like behaviors (10-13). However, details regarding the mechanisms of these neurological processes involved in pathological damage in depression are not fully understood. Therefore, it is necessary to achieve a more comprehensive identification of potential molecular targets and novel pathways underlying the genesis and development of depression.

Recent genomic studies have revealed that dysregulated expression within a broad spectrum of noncoding RNAs (ncRNAs) is implicated in various neurological diseases (14). MicroRNAs (miRNAs), which are widespread and diverse

Conflict of interest: The authors have declared that no conflict of interest exists. Copyright: (5) 2021, American Society for Clinical Investigation.

Submitted: February 18, 2021; Accepted: July 1, 2021; Published: August 16, 2021.

Reference information: J Clin Invest. 2021;131(16):e148853.

https://doi.org/10.1172/JCl148853. endogenous ncRNAs that can regulate gene expression by directly modifying messenger RNA (mRNA) after transcription (15), have attracted considerable attention of late because of their capacity to regulate neuronal development and function $(16,17)$. Through their ability to target and suppress endogenous mRNAs, miRNAs can inhibit the translation of proteins (18). Therefore, any disruption or imbalance in the expression and function of miRNA networks could lead to neurological diseases and, in fact, there is accumulating evidence that miRNAs are involved in neurological disorders such as Parkinson's disease (PD), Alzheimer's disease (AD), and major depressive disorder (MDD) (19-21). Thus, expression changes in specific miRNAs could serve as potential biomarkers for diagnostic or therapeutic targets in clinical practice (22). However, the mechanisms underlying miRNAs' contribution to the development and progression of these neurological disorders, particularly depression, remain largely unknown. Thus, the screening of specific miRNAs, their underlying mechanisms, and possible downstream signaling pathways related to neuronal dysfunction associated with depression may provide new therapeutic strategies for the treatment of this condition.

In the present study, we examined the differentially expressed miRNA profile in a chronic unpredictable mild stressinduced (CUMS-induced) rat model of depression versus normal controls using a high-throughput microarray. We found that miR-26a-3p, an miRNA that is potentially responsible for regulation of mRNA-encoding proteins related to stress and depression (23), showed significantly differential expression within the hippocampal dentate gyrus (DG) of CUMS versus control rats and interacted with the phosphate and tension homology deleted on chromosome ten (PTEN)/phosphoinositide 3-kinase 
A
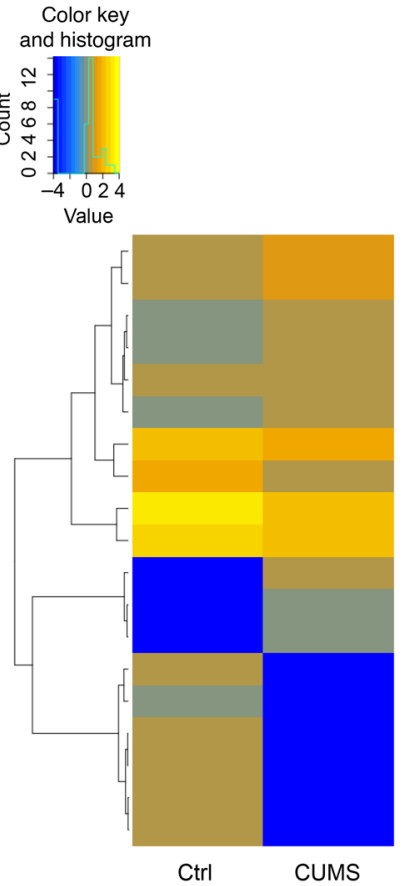

D
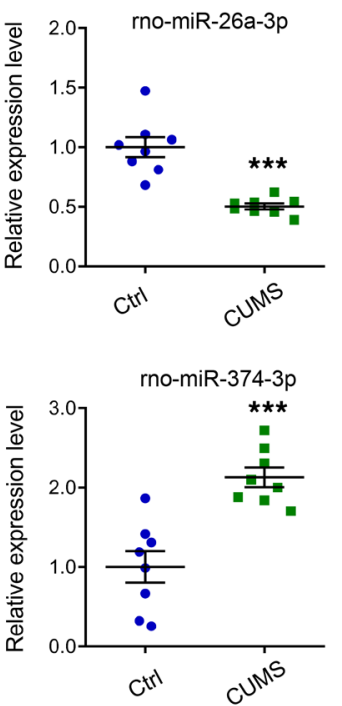

rno-miR-374-3p rno-miR-539-3p rno-miR-335 rno-miR-219a-5p rno-miR-190-5p rno-miR-376c-3p rno-miR-34b-3p rno-miR-448-3p rno-miR-1298 rno-miR-221-5p rno-miR-3084c-5p rno-miR-3541 rno-miR-144-5p rno-miR-500-3p rno-miR-26a-3p rno-miR-223-3p rno-miR-1912-3p rno-miR-152-5p rno-miR-483-5p
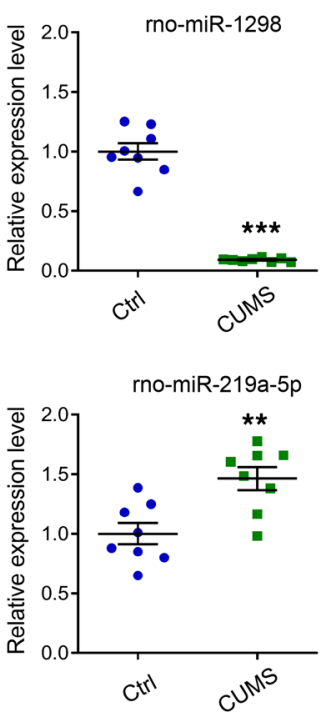

B

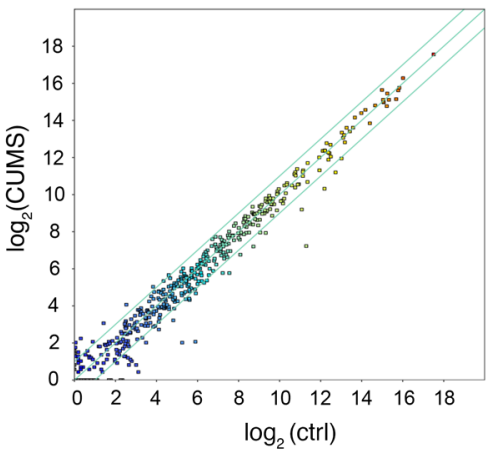

C

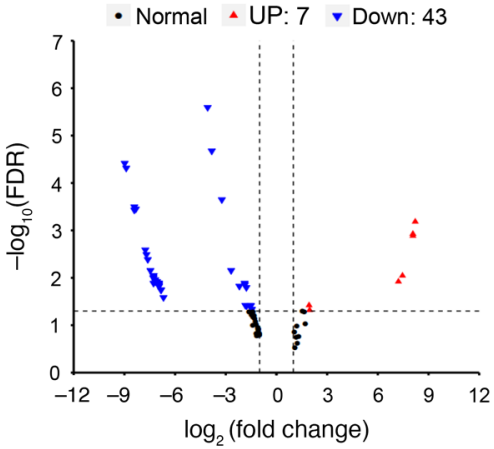

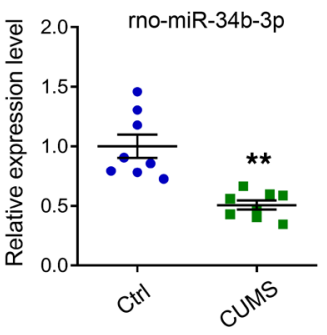

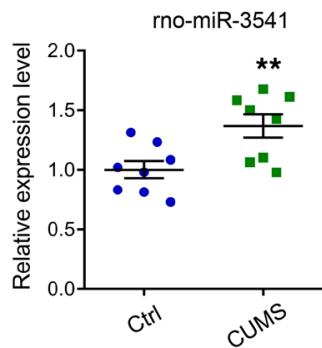

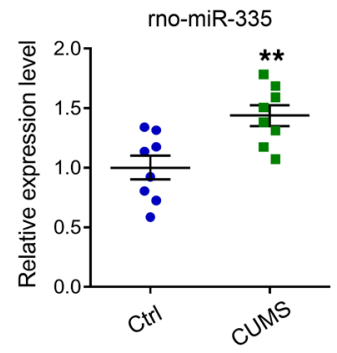

Figure 1. miRNA expression profiles of DG tissue derived from control and CUMS-induced depression groups. (A) Representative heatmap of differential miRNA expression levels obtained by sequencing on the Illumina HiSeq 2500 platform. $n=3$ rats per group. (B) Scatter plots were used to evaluate differences in the expression of miRNAs between the 2 groups. The miRNAs above the top green line and below the bottom green line indicate a greater than 2.0 -fold change between the 2 groups. $n=3$ rats per group. (C) Volcano plot indicating differential expression between the 2 groups. $P$ less than 0.05 and fold change greater than 2 were considered statistically significant. $n=3$ rats per group. (D) The expression levels of 8 miRNAs were validated by qPCR in DC tissues. $n=8$ rats per group. Experiments were performed in triplicate with 3 biological replicates for all panels. Data are presented as mean \pm SEM. ${ }^{* *} P<0.01,{ }^{* * *} P<0.001$ vs. control by Student's $t$ test. Ctrl, control.

$(\mathrm{PI} 3 \mathrm{~K}) /$ protein kinase B (Akt) signaling pathway. Complementing these findings, the knockdown of miR-26a-3p in DG regions of control rats accelerated neuronal deterioration and induced depression-like behaviors, whereas upregulation of miR-26a-3p within DG regions of CUMS-induced depressed rats rescued neuronal deterioration and depression-like phenotypes. Accordingly, these results provide insights into mechanisms involving the functional regulation of miRNAs in depression, and specif- ically, identify miR-26a-3p as a potential target for depression prognosis and therapy.

\section{Results}

Identification of differentially expressed miRNA profiles in the CUMS-induced rat model of depression. miRNA expression profiles vary markedly as a function of different cell types and conditions. Therefore, we first investigated miRNA expression profiles within 
A
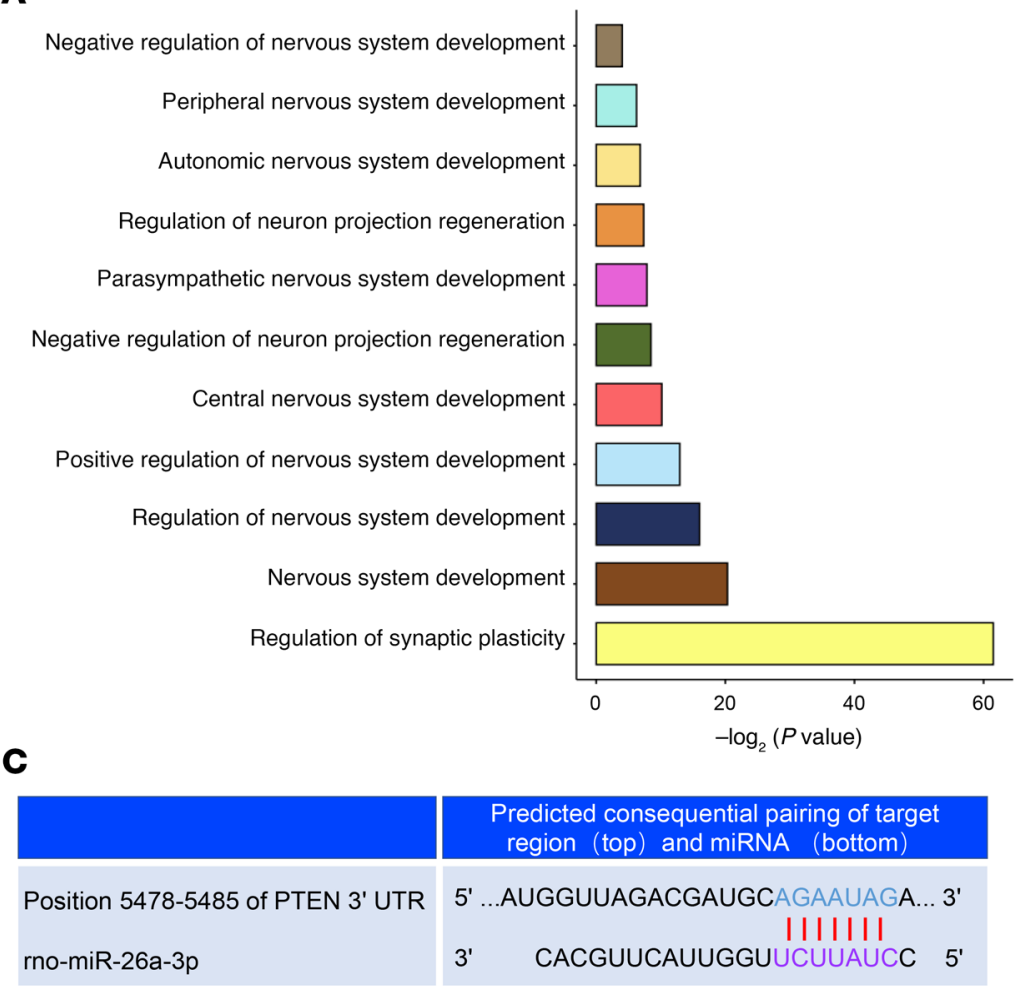

B
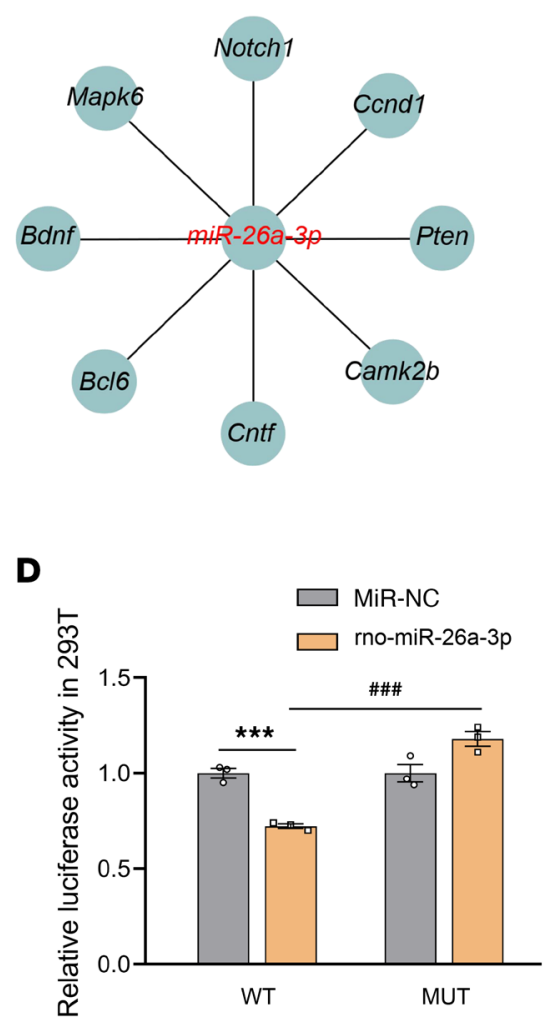

Figure 2. Prediction and validation of target genes of miR-26a-3p and its signaling pathways. (A) DAVID functional annotation for the miR-26a-3p target genes with horizontal axes showing - $\log _{2}$-transformed $P$ values. (B) Bioinformatics prediction by DIANA-miRPath of 8 target genes of miR26a-3p that appear to be related to pathways in depression. (C) Putative seed-matching sites between miR-26a-3p and PTEN. (D) Dual-luciferase reporter assay was performed to detect relative luciferase activities of WT and MUT PTEN reporters. $n=3$ per group. Experiments repeated at least 3 times. Data are presented as mean \pm SEM. ${ }^{* * *} P<0.001$ vs. WT + miR-NC; ${ }^{\# \# ~} P<0.001$ vs. WT + rno-miR-26a-3p by 1 -way ANOVA with post hoc Tukey's correction. MUT, mutated.

he hippocampal DG in normal control and CUMS-induced depressed rats using the Illumina HiSeq 2500 high-throughput sequencing (miRNA-seq) technique. Expression patterns of miRNAs were compared between depressed and normal rats using hierarchical clustering analysis (Figure 1A). Differentially expressed miRNAs are displayed after fold change filtering (Figure $1 B$ ), with statistically significant differential changes in miRNAs between the two groups identified with volcano plot filtering (Figure 1C) and principal component analysis (PCA) (Supplemental Figure 1; supplemental material available online with this article; https://doi.org/10.1172/JCI148853DS1). With this analysis, 57 miRNAs were found to be significantly different between depressed and normal DG samples using a fold change of 2.0 or greater and $P$ less than 0.05 as the cutoff threshold. Among the differentially expressed miRNAs, 9 were upregulated and 48 downregulated in DG regions of depressed as compared with normal rats. These results demonstrate that clear differences are present in the differential expression of miRNAs in DG tissues between normal and depressed rats. Next, to validate miRNA-seq results for some of these miRNAs, levels of miR-26a-3p, miR-1298, miR211-5p, and miR-34b-3p in DG tissues, including the samples for sequencing analysis, were determined using quantitative PCR (qPCR). These 4 miRNAs showed significantly decreased levels within DG regions of CUMS-induced versus normal rats (Figure
1D). These results indicate that the real time qPCR data were consistent with that of the miRNA-seq analysis regarding expression levels of these 4 miRNAs.

PTEN is a direct target gene of miR-26a-3p. To examine the functional effects of miRNAs, those that were possibly associated with depression-related pathways were assessed. All candidate miRNAs involved in possible pathways, as identified using a $P$-value cutoff of 0.05 , were determined using DIANA-miRPath (24). With this analysis, miR-26a-3p was recognized as showing the greatest potential for being implicated in neuronal injuryrelated pathways, which could then be considered as contributing to the pathogenesis of depression. Therefore, we focused our investigation on miR-26a-3p, which was significantly decreased to the lowest levels as observed in the DG of these depressed rats. The predicted target genes of miR-26a-3p, as associated with neuronal regulatory pathways, were the examined using DIANAmiRPath (Figure 2A). The functional annotation data revealed that 8 genes, which were significantly correlated with neuronal plasticity and development, could be regulated by miR-26a-3p (Figure 2B). Among these predicted target genes, PTEN, which contributes to cell development, migration, and apoptosis, was considered as a crucial factor involved in mediating the regulatory effects of miR-26a-3p related to neuronal injury in depression. Therefore, to determine the molecular mechanisms of miR-26a-3p, a dual- 
A

CMV EGFP $-\begin{aligned} & \text { Scramble } \\ & \text { sequence }\end{aligned}$ WPRE

miR-26a-sponge

CMV eGFP — miR-26a-sponge WPRE

B

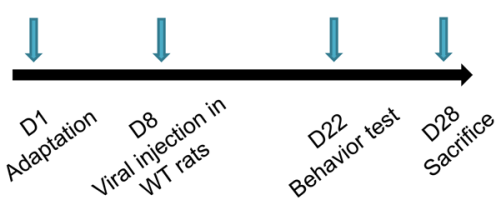

C

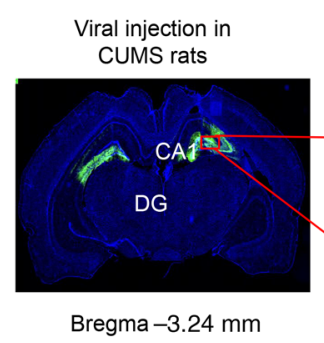

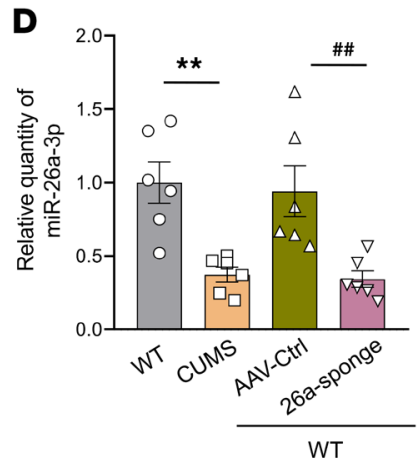

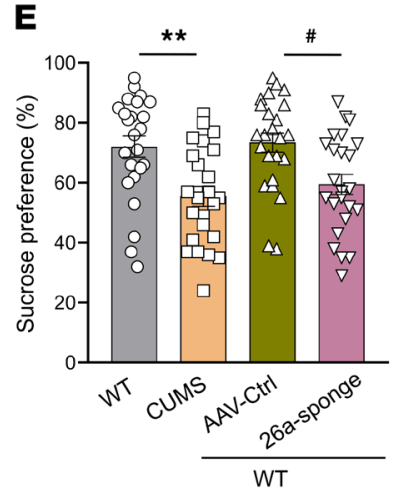

G

WT

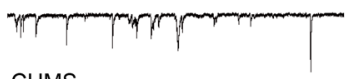

CUMS

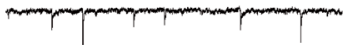

WT/AAV-Ctrl

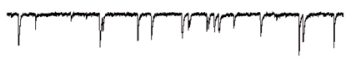

WT/26a-sponge
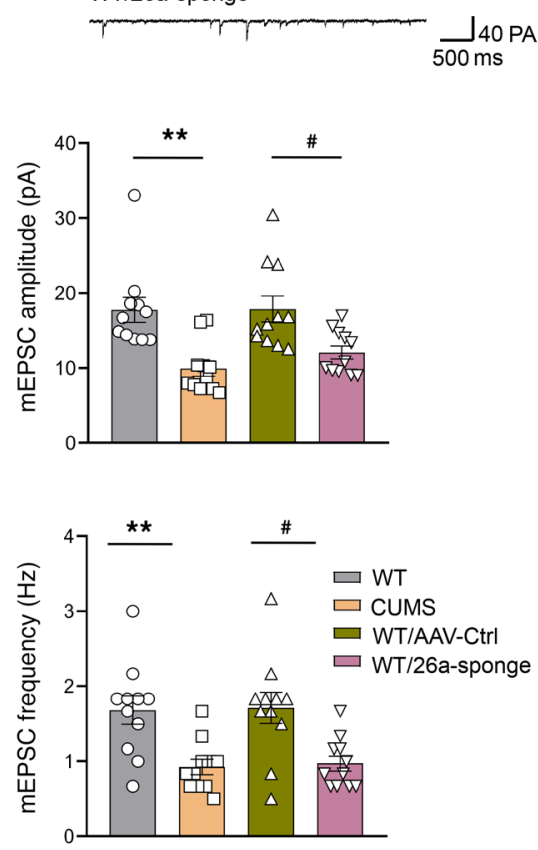

H
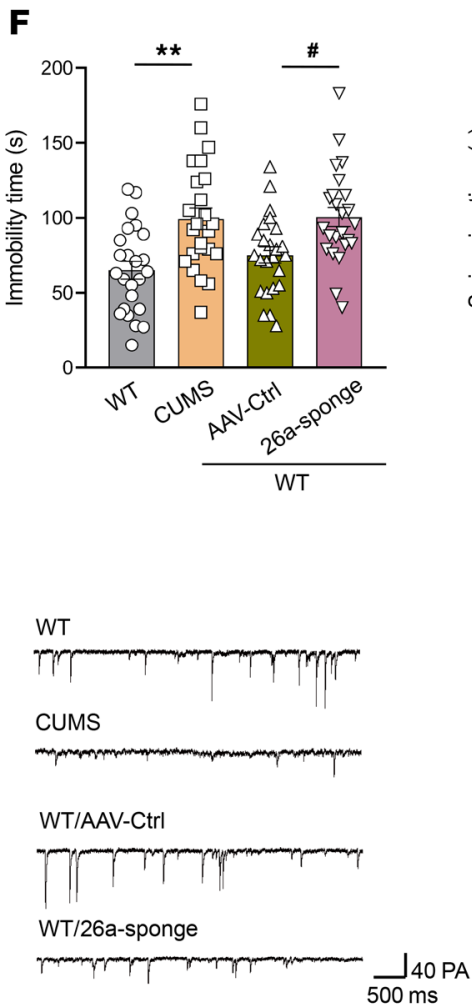

I

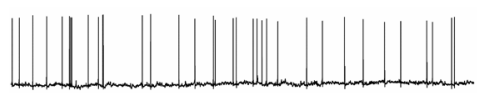

WT
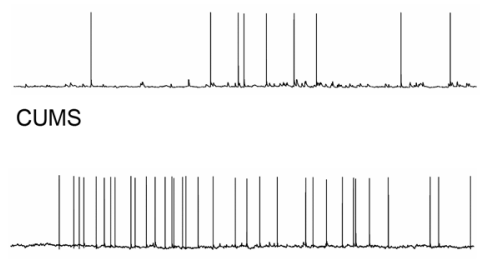

WT/AAV-CtrI

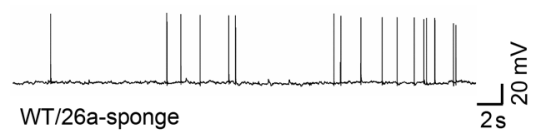

$\square$ WT

$\square$ CUMS

$\square$ WT/AAV-Ctrl

$\square$ WT/26a-sponge

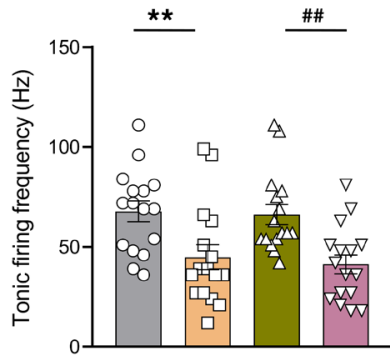


Figure 3. Knockdown of miR-26a-3p within the DG induced depression-like behaviors in normal rats. (A) Schematic of AAV vectors engineered to knock down miR-26a-3p or a vector control construct. (B) Experimental paradigm for virus injection and behavioral testing. D, day. (C) Illustration of bilateral virus injection site in the DC hippocampus. Scale bar: $20 \mu \mathrm{m}$. (D) Quantitative real-time PCR was used to validate the efficiency of miR-26a-3p knockdown. $n=6$ rats per group. Three independent biological replicate experiments were performed for each group. (E) Knockdown of miR-26a-3p within the DC decreased sucrose consumption in the sucrose preference test and $(\mathbf{F})$ increased immobility times and decreased swimming times of rats in the forced-swim test. $n=18$ rats per group for behavioral test. Knockdown of miR-26a-3p in DG neurons produced changes in (G) mEPSCs, (H) sEPSCs, and (I) spontaneous burst activity. $n=10$ cells from 6 rats per group in $\mathbf{G}$ and $\mathbf{H} ; n=16$ cells from 6 rats per group in I. Electrophysiological recordings were repeated in at least 3 independent experiments. Data are presented as mean \pm SEM. ${ }^{* *} P<0.01$ vs. WT; ${ }^{\#} P<0.05,{ }^{\#} P<0.01$ vs. AAV-control (WT + AAV-control) by 1-way ANOVA with post hoc Tukey's correction. Ctrl, control.

luciferase reporter assay was used to validate the direct target gene of miR-26a-3p. It was predicted that the 3'-UTR of PTEN mRNA would contain a putative binding site for the seed-match sequence of miR-26a-3p (Figure 2C). Results of this assay demonstrated that miR-26a-3p significantly repressed reporter activity of the transcript containing the wild-type (WT) 3'-UTR of PTEN mRNA (by $0.32 \pm 0.02$-fold versus a negative control miRNA [miR-NC], $P<$ 0.01 ), indicating that miR-26a-3p exerts a direct regulatory effect on PTEN (Figure 2D).

Knockdown of miR-26a-3p in the DG induces depression-like behaviors and decreased synaptic transmission in rats. Oligonucleotides against miR-26a-3p were cloned into an adeno-associated virus (AAV) vector (AAV-miR-26a-3p-sponge) to knock down miR-26a-3p expression and function in the DG of normal rats, while another vector including a scrambled sequence was constructed to serve as a negative control (Figure 3A and Supplemental Figure 2). After bilateral stereotaxic infusion into the DG (Figure 3, B and C), the knockdown efficiency of miR-26a-3p was examined by qPCR and showed a $74 \%$ decrease in DG regions compared with the control group $(P<0.01)$, a trend similar to that observed in the CUMS group (Figure 3D). Particularly significant was the observation of depression-like behaviors in these miR-26a-3p-knockdown rats 14 days after infusion. Results from a sucrose preference test showed that knockdown of miR-26a$3 p$ within the DG significantly decreased the consumption of sucrose as compared with rats receiving a mock control injection, results that provide an index of anhedonia (Figure 3E). Moreover, increased immobility and decreased swimming times in rats with knockdown of miR-26a-3p were observed in a forcedswim test, responses indicative of behavioral despair in these rats (Figure $3 \mathrm{~F}$ ). In addition, results from the open-field test showed that knockdown of miR-26a-3p had no effect on the spontaneous locomotor activity of rats ( $P>0.05$; Supplemental Figure 3$)$. Next, whole-cell patch-clamp recordings in neurons from DG coronal slices and miniature and spontaneous excitatory postsynaptic currents (mEPSCs and sEPSCs, respectively) were assessed in these rats. We found that, as compared with controls receiving the mock empty vector, the knockdown of miR-26a-3p inhibited both the amplitudes and frequencies of mEPSCs and sEPSCs (Figure 3, G and $\mathrm{H}$ ) as well as spontaneous tonic firing (Figure $3 \mathrm{I}$ ), effects sim- ilar to that observed in CUMS-exposed rats. These results suggest that a deficit in miR-26a-3p inhibits synaptic transmission in DG pyramidal neurons in the hippocampal microcircuit, which may then contribute to the depression-like behaviors observed in rats.

Knockdown of miR-26a-3p increases PTEN expression and inhibits autophagy in rats. Results from Western blot analysis showed that, in contrast to that observed in mock controls, the knockdown of miR-26a-3p increased protein levels of PTEN in the DG region, again findings that were similar to the increased PTEN expression levels seen in rats following 5 weeks of CUMS exposure (Figure 4A). Moreover, expression of PI3K and phosphorylated Akt was significantly downregulated, accompanied by an upregulation of p53 in the miR-26a-3p-knockdown group as compared with controls (Figure 4A). To further explore possible neuronal mechanisms of miR-26a-3p in depression, we examined whether this miR-26a-3p/PTEN axis could modulate autophagy. We found that knockdown of miR-26a-3p in DG regions also markedly downregulated LC3-II/LC3-I and beclin-1 expression, while it increased the expression of p62 (Figure 4B). Meanwhile, images from electron microscopy revealed that the amount of autophagosomes in the DG region were significantly decreased (Figure 4C). These results demonstrate that PTEN is a target of miR-26a$3 p$ and the upregulation of PTEN expression by the knockdown of miR-26a-3p appears to result in suppression of autophagic activity within the DG.

Knockdown of miR-26a-3p induces dysregulation of neuronal plasticity in the DG. Results from our immunofluorescence analysis also showed that miR-26a-3p-knockdown rats exhibited a significant decrease in key neuroplasticity-related markers, including synaptophysin (Syn) and postsynaptic density protein 95 (PSD-95) as compared with the mock controls (Figure 5A), and as well as microtubule-associated protein 2 (MAP-2) (Supplemental Figure 4, A and B). Results from our Western blot analysis revealed that low levels of expression in a series of neuroplasticityrelated regulators within the DG were observed in response to miR-26a-3p knockdown as compared with that in mock control rats (Figure 5B). These findings from miR-26a-3p-knockdown rats were similar to those found within the DG of our CUMSinduced rat model of depression. Moreover, the changes in mRNA expression level of these main neuroplasticity-related regulators in response to miR-26a-3p knockdown were similar to the changes in their protein expression levels (Supplemental Figure 4C). Morphological examination revealed that decreased numbers of synapses were present within DG regions in both miR-26a-3p-knockdown and CUMS-induced depressed rats (Supplemental Figure 4D). Dysregulation in neuroplasticity was further confirmed by the significant losses of dendritic spines observed in miR-26a-3p-knockdown rats (Figure 5C).

Knockdown of miR-26a-3p promotes neuronal apoptosis in the DG. Results from immunofluorescent staining showed that cleaved caspase-3, a terminal regulator that prompts the apoptotic process, was significantly increased (Figure 6A), while the neuronal precursor marker, doublecortin X (DCX) (Figure 6A), and neural stem cell marker, nestin (Supplemental Figure 5, A and B), were significantly decreased within DG regions of miR-26a-3pknockdown rats. Following miR-26a-3p knockdown, significant increases in protein levels of the proapoptotic factors, Bax, 

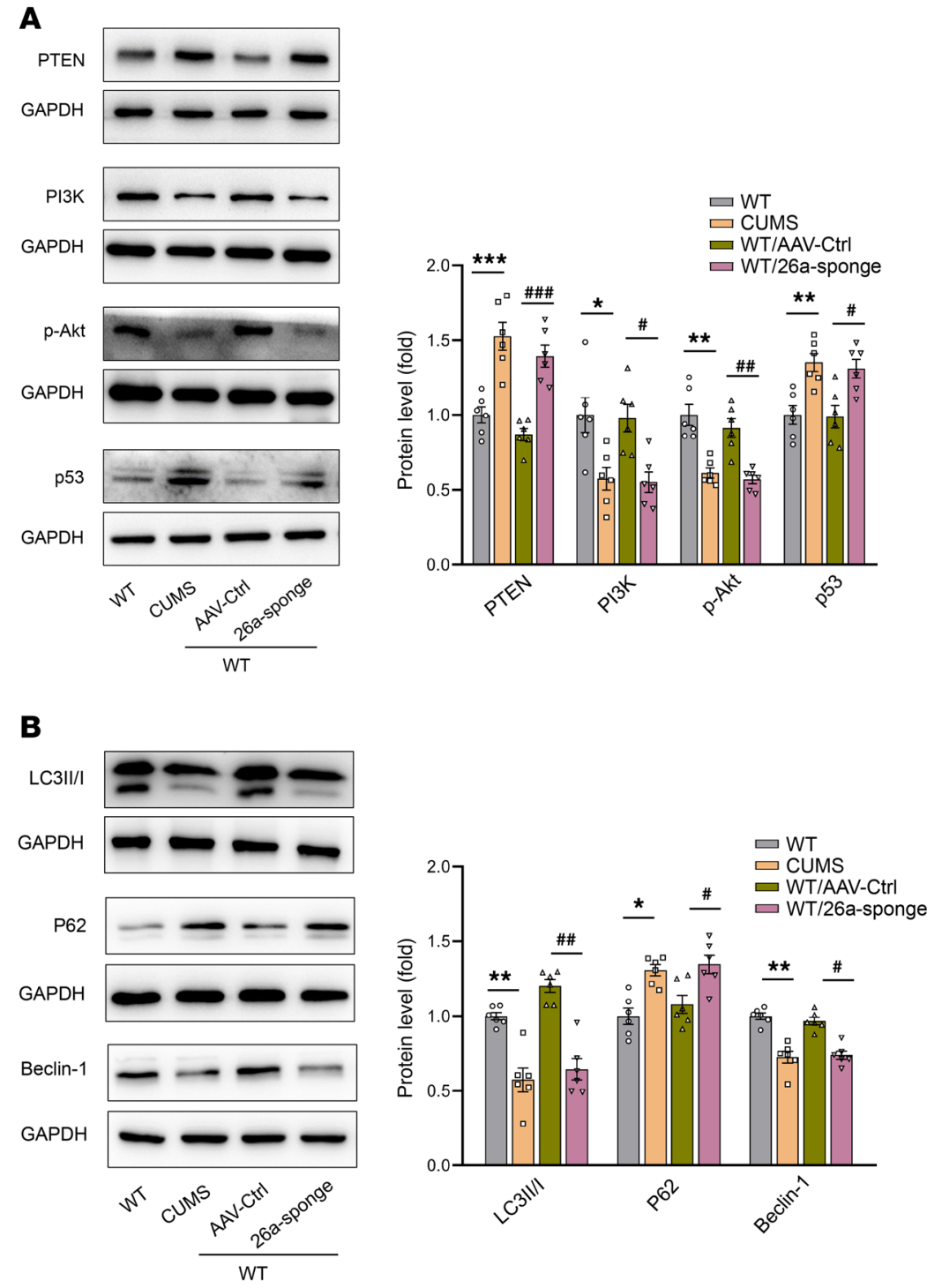

\section{C}
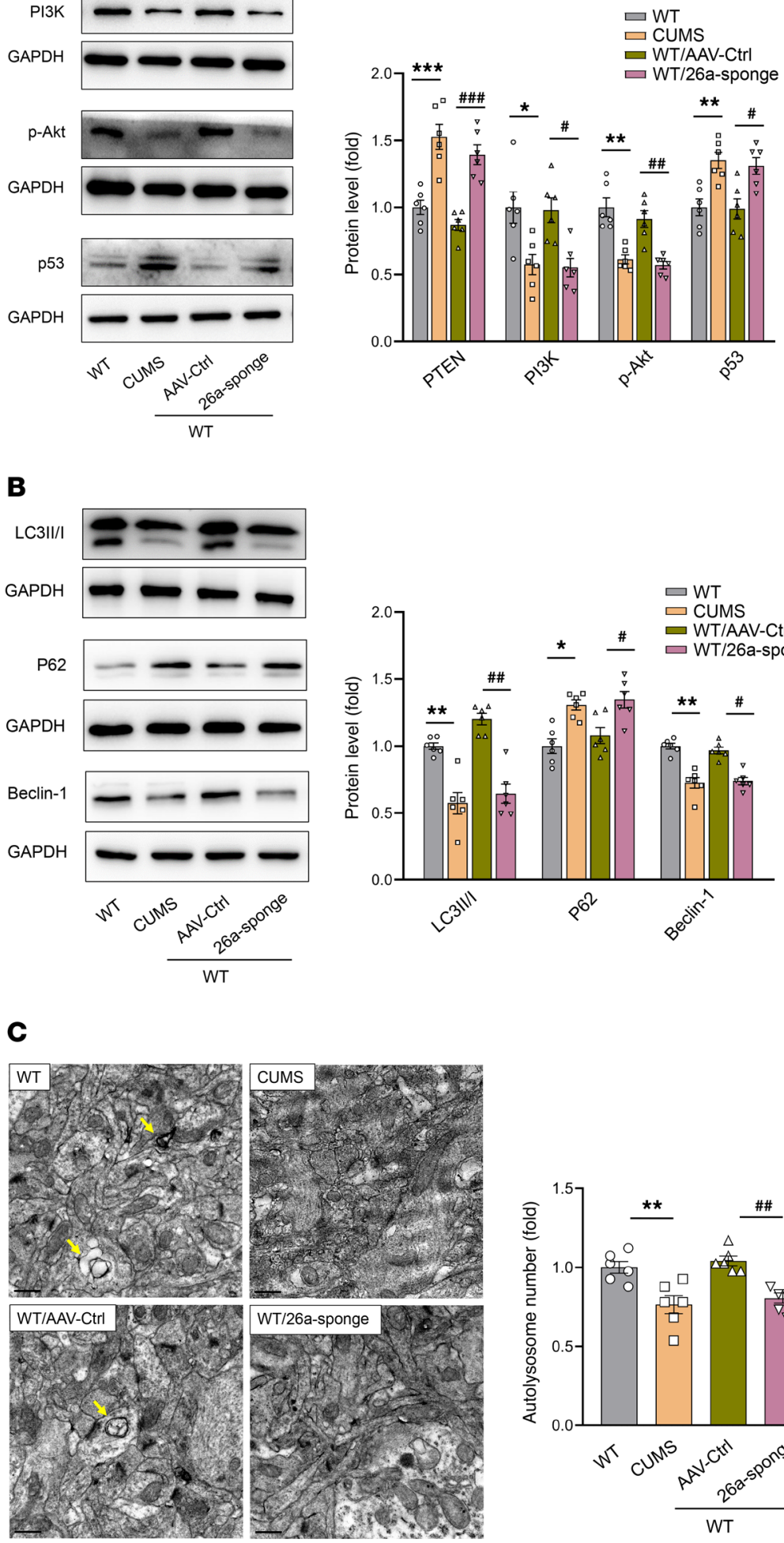

caspase-3, and caspase-9, accompanied by decreased expression levels of $\mathrm{Bcl}-2$ were observed within the DG, as indicated from results of Western blotting (Figure 6B). Similar trends were obtained with regard to transcriptional levels of these apoptosis-related factors (Supplemental Figure 5C). Morphological changes, as observed with Hoechst-33258 staining (Supplemental Figure 5D) and transmission electron microscopy (Figure 6C), demonstrated that the nuclei within DG neurons exhibited remarkable characteristics of apoptosis, including nuclear chromatin margination, aggregation, and condensation in miR-26a-3p-knockdown versus mock control rats. The number of apoptotic cells within DG areas was correspondingly increased after knockdown of miR-26a-3p. Interestingly, we consistently observed that these tendencies for apoptotic morphological changes and apoptosis-related factor expression, as observed in miR-26a-3p-knockdown rats, were also present in CUMS rats. These results provide further evidence suggesting that downregulation of miR-26a-3p within the DG contributes to neuronal apoptosis, which may then be responsible for the induction of neuronal injury and depression-like behaviors in rats.

Overexpression of $m i R-26 a-3 p$ in the DG rescues depression-like behaviors and impaired synaptic transmission in depressed rats. As a complementary approach to evaluate the role of miR-26a-3p in maintaining homeostasis and function of the DG neuronal network, an AAV-miR-26a-3p vector was infused into the $\mathrm{DG}$ region to overexpress miR-26a-3p in CUMS rats (Figure 7A). A presentation of the experimental design is shown in Figure 7B. The infection efficiency of miR-26a$3 \mathrm{p}$ was examined by immunofluorescence (Figure 7C) and a significant increase in miR-26a-3p expression levels within isolated DG regions was observed in miR-26a-3p-overexpressing rats (Figure 7D). Interestingly, restoration of miR-26a-3p 
A

Syn
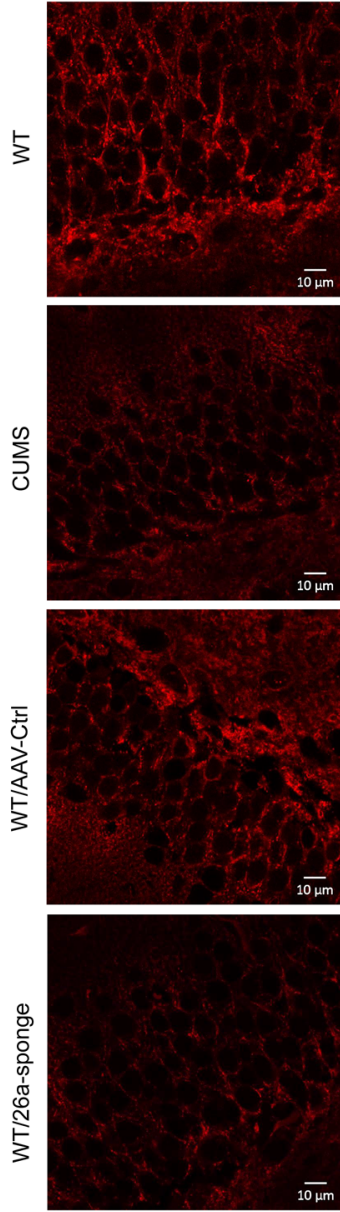

B
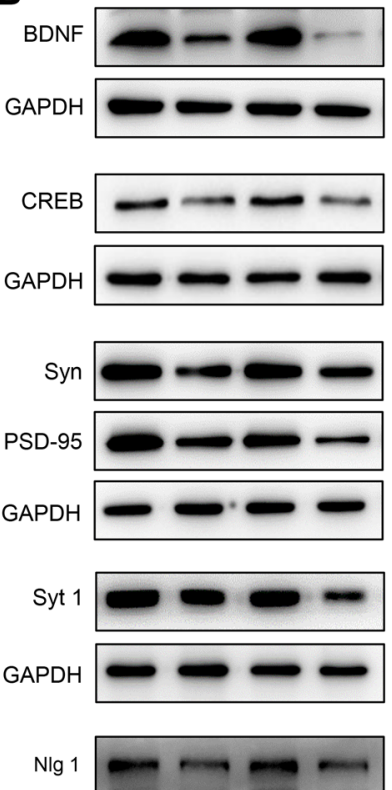

GAPDH

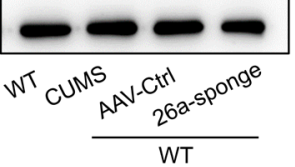

Syn/DAPI

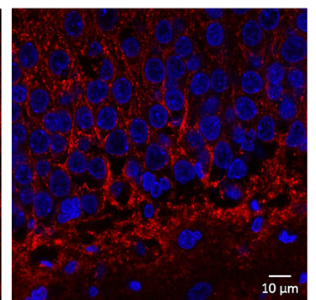

$\overline{0 \mu \mathrm{m}}$
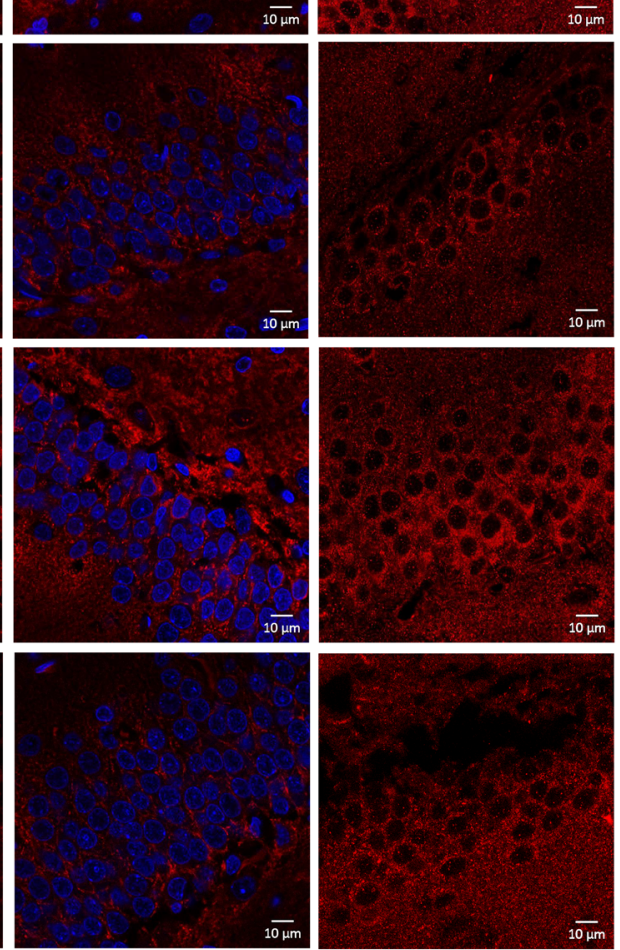

c
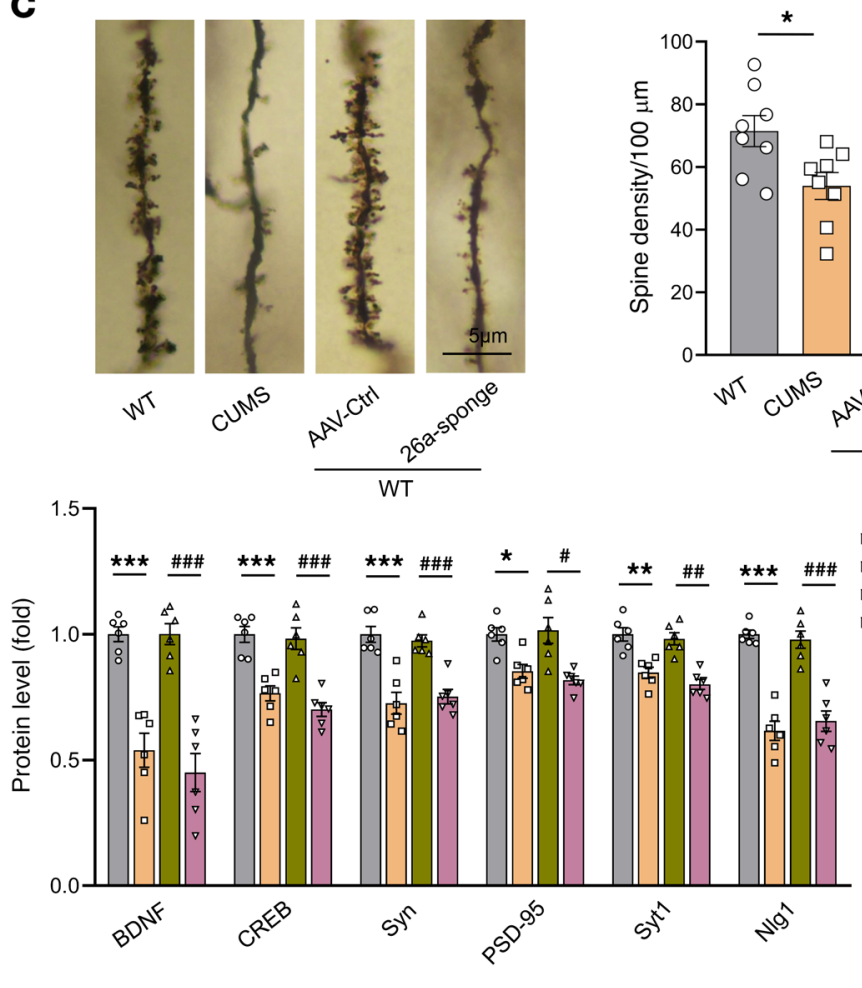

PSD-95/DAPI
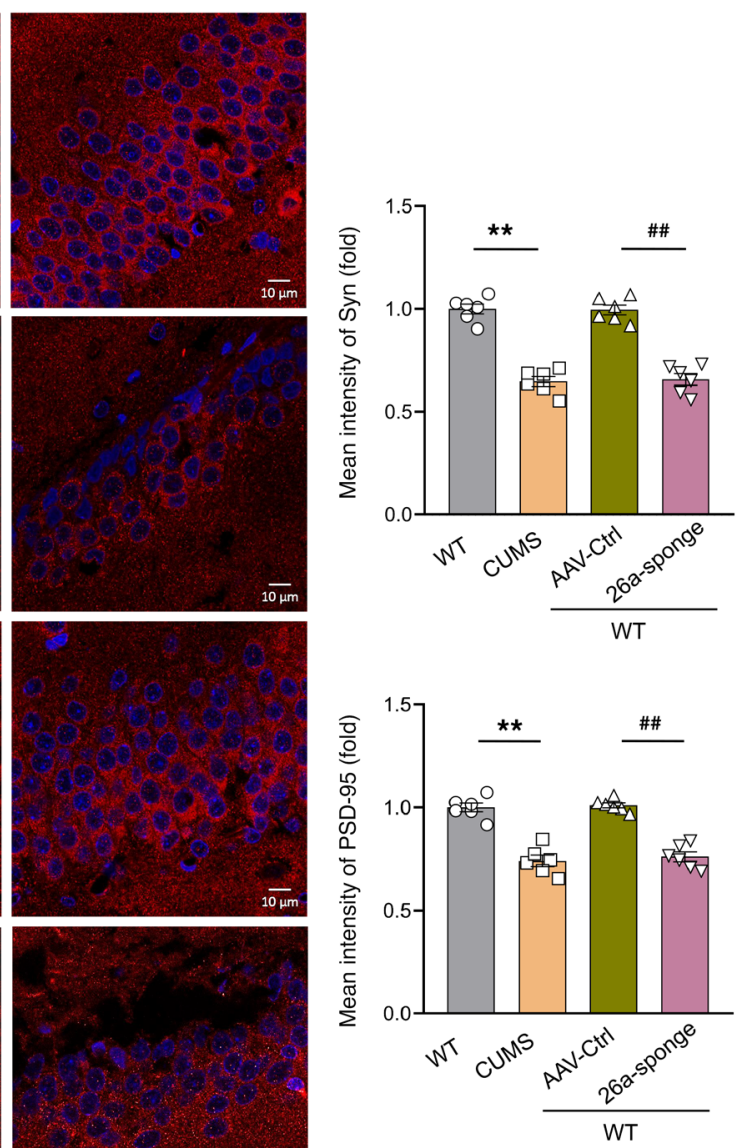
Figure 5. Knockdown of miR-26a-3p within the DC of normal rats induced dysregulation of neuroplasticity. (A) Representative confocal microscopic images showing expression of Syn and PSD-95 within the DG of different groups. Scale bars: $10 \mu \mathrm{m} . n=6$ rats per group and at least 4-6 images from 1 animal. (B) Knockdown of miR-26a-3p decreased protein levels of neuroplasticity-related mediators in the DC. $n=6$ rats per group. Western blotting results for Syn and PSD-95 were from the same samples and run in parallel in different gels. Three independent biological replicate experiments were performed. (C) Representative Golgi staining images and summary of data showing dendritic spines in DG neurons of different groups. Scale bar: $5 \mu \mathrm{m} . n=8$ rats per group and at least 5 pyramidal neurons from 1 animal. Immunofluorescence and Golgi staining were repeated at least 3 times and quantitation was done for representative samples from each group. Data are presented as mean \pm SEM. ${ }^{*} P<0.05$, ${ }^{*} P<$ $0.01,{ }^{* *} P<0.01$ vs. WT; ${ }^{\# P}<0.05,{ }^{\# \# P}<0.01,{ }^{\# \#} P<0.001$ vs. AAV-control (WT + AAV-control) by 1-way ANOVA with Tukey's post hoc correction. Ctrl, control; Syt1, synaptotagmin 1; Nlg1, neuroligin 1.

levels within the DG significantly ameliorated symptoms of anhedonia and behavioral despair resulting from 5 weeks of CUMS exposure, as evidenced by increases in sucrose consumption (Figure 7E) and decreases in immobility times and increases in swimming times (Figure 7F) as compared with CUMS rats receiving the mock virus. However, results from the open-field test showed that overexpression of miR-26a-3p within DG regions of CUMS rats had no effect on the spontaneous locomotor activity of rats $(P>$ 0.05; Supplemental Figure 6). We also found that overexpression of miR-26a-3p in CUMS rats significantly increased the amplitudes and frequencies of both mEPSCs and sEPSCs in DG pyramidal neurons (Figure 7, G and $\mathrm{H}$ ) as well as spontaneous tonic activity (Figure 7I) in the CUMS group. These findings suggest that restoration of abnormal excitatory synaptic activity in DG granule cells contributes to the amelioration of depression-like behavior in CUMS rats and that upregulation of miR-26a-3p within the DG rescues the core symptoms of depression in depressed rats.

Overexpression of miR-26a-3p suppresses PTEN expression and increases autophagy in depressed rats. Overexpression of miR-26a3 p within the DG significantly decreased protein expression levels of PTEN, a direct target of miR-26a-3p, in depressed rats (Figure $8 \mathrm{~A}$ ), and the changes in protein levels of PI3K, phosphorylated Akt, and p53 resulting from CUMS exposure were also restored in response to the overexpression of $\mathrm{miR}-26 \mathrm{a}-3 \mathrm{p}$ within the DG. Moreover, we observed that overexpression of miR-26a-3p increased levels of LC3-II/LC3-I and beclin-1 and decreased levels of p62 (Figure $8 \mathrm{~B}$ ). The expression changes in these autophagyrelated proteins provide further support for the hypothesis that the miR-26a-3p/PTEN pathway could rescue depression-like behaviors via regulating autophagic activity in depressed rats. Finally, electron microscopic images revealed the restoration in autophagy resulting from upregulation of miR-26a-3p within the DG of depressed rats (Figure $8 \mathrm{C}$ ). Taken together, these results provide compelling evidence indicating that miR-26a-3p induces autophagy within the DG of depressed rats.

Overexpression of miR-26a-3p ameliorates the dysregulation of neuronal plasticity in depressed rats. Results from immunofluorescence analysis showed that the main synaptic markers, Syn, PSD-95 (Figure 9A), and MAP-2 (Supplemental Figure 7, A and $\mathrm{B})$, were increased within the DG area following miR-26a-3p over- expression. Overexpression of miR-26a-3p, as achieved with an AAV-miR-26a-3p vector, markedly ameliorated the dysregulation of neuronal plasticity in depressed rats as evidenced by increased protein (Figure 9B) and mRNA (Supplemental Figure 7C) levels of plasticity-related mediators within the DG of depressed rats. Moreover, we observed that the overexpression of miR-26a-3p significantly restored the number of synapses (Supplemental Figure 7D) and dendritic spine densities (Figure 9C) in DG neurons, which had been reduced by CUMS exposure. These results provide convincing evidence that overexpression of miR-26a-3p ameliorated the dysregulation of neuroplasticity in depressed rats.

Overexpression of miR-26a-3p inhibits neuronal apoptosis in depressed rats. Compared with the nonstressed control group, CUMS rats displayed decreased expression of the neurogenesis markers, DCX (Figure 10A) and nestin (Supplemental Figure 8, A and B), along with increased protein (Figure 10B) and mRNA (Supplemental Figure 8C) expression levels of proapoptotic factors within DG areas. All these changes observed in these depressed rats were alleviated with upregulation of miR-26a-3p. Moreover, morphological results obtained with Hoechst-33258 staining (Supplemental Figure 8D) and transmission electron microscopy (Figure 10C) showed that upregulation of miR-26a-3p within the DG of depressed rats significantly restored the nuclear deterioration resulting from CUMS exposure. These results suggest that miR-26a-3p suppresses neuronal apoptosis and promotes neurogenesis within the DG area, effects that may work through the PTEN signaling pathway in depression.

PTEN mediates neuronal and behavioral anomalies resulting from miR-26a-3p deficits in the DG. Finally, to substantiate the hypothesis that the neuronal deterioration induced by miR26a-3p was dependent on the PTEN signaling pathway, miR26a-3p-knockdown rats were treated with the PTEN inhibitor, dipotassium bisperoxo(pyridine-2-carboxyl)oxovanadate $[\mathrm{bpV}(\mathrm{pic})]$. As shown in Figure $11 \mathrm{~A}, \mathrm{bpV}$ (pic) treatment $(0.2 \mathrm{mg} /$ $\mathrm{kg}$, i.p.) markedly upregulated PI3K and induced Akt phosphorylation, two vital downstream components of the PTEN pathway that were significantly suppressed by miR-26a-3p knockdown in $\mathrm{DG}$ regions. $\mathrm{bpV}$ (pic) treatment also decreased the upregulation of p53 resulting from this miR-26a-3p deficit and markedly increased LC3-II/LC3-I and beclin-1 expression and decreased levels of p62 (Figure 11B), suggesting that inhibition of PTEN restored the autophagy suppressed by the miR-26a-3p deficit. In addition, $\mathrm{bpV}$ (pic) treatment upregulated neuroplasticityrelated mediators (Figure 11C) and reduced expression of proapoptotic factors in miR-26a-3p-knockdown rats (Figure 11D). These results demonstrated that this pharmacological inhibition of PTEN significantly rescued the neuronal deterioration and cell death resulting from miR-26a-3p knockdown. $b p V(p i c)$ treatment also effectively ameliorated the depression-like symptoms from this miR-26a-3p deficit, as based on results obtained with the sucrose preference (Figure 11E) and forced-swim (Figure 11F) tests and reversed the decreases in frequencies of spontaneous tonic firing (Figure 11G) in DG neurons of depressed rats.

\section{Discussion}

Despite accumulating evidence from increasing numbers of studies indicating a relationship between depression and neuro- 
A
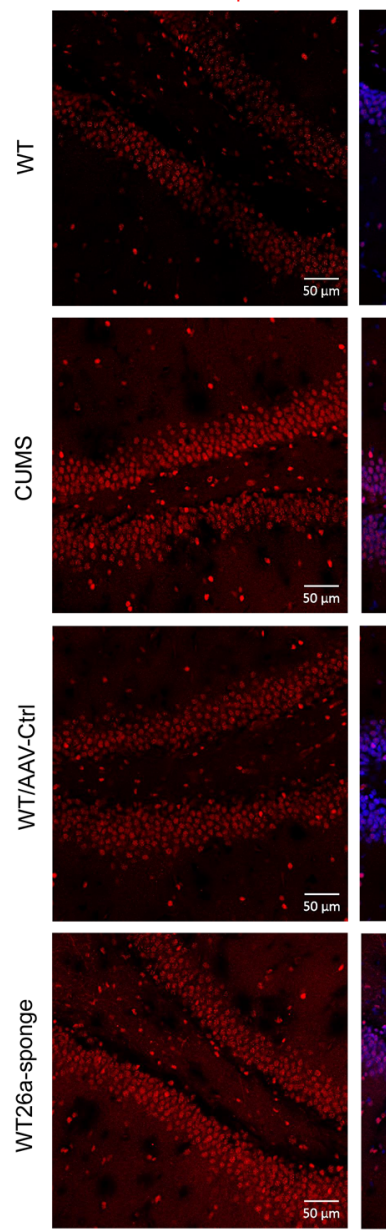

B

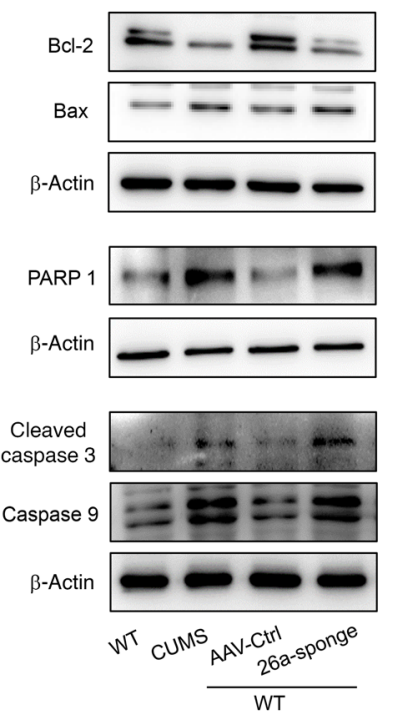

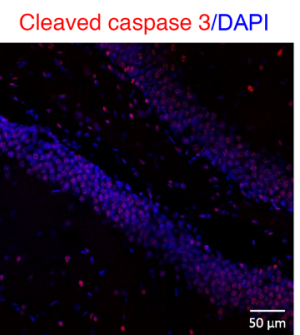
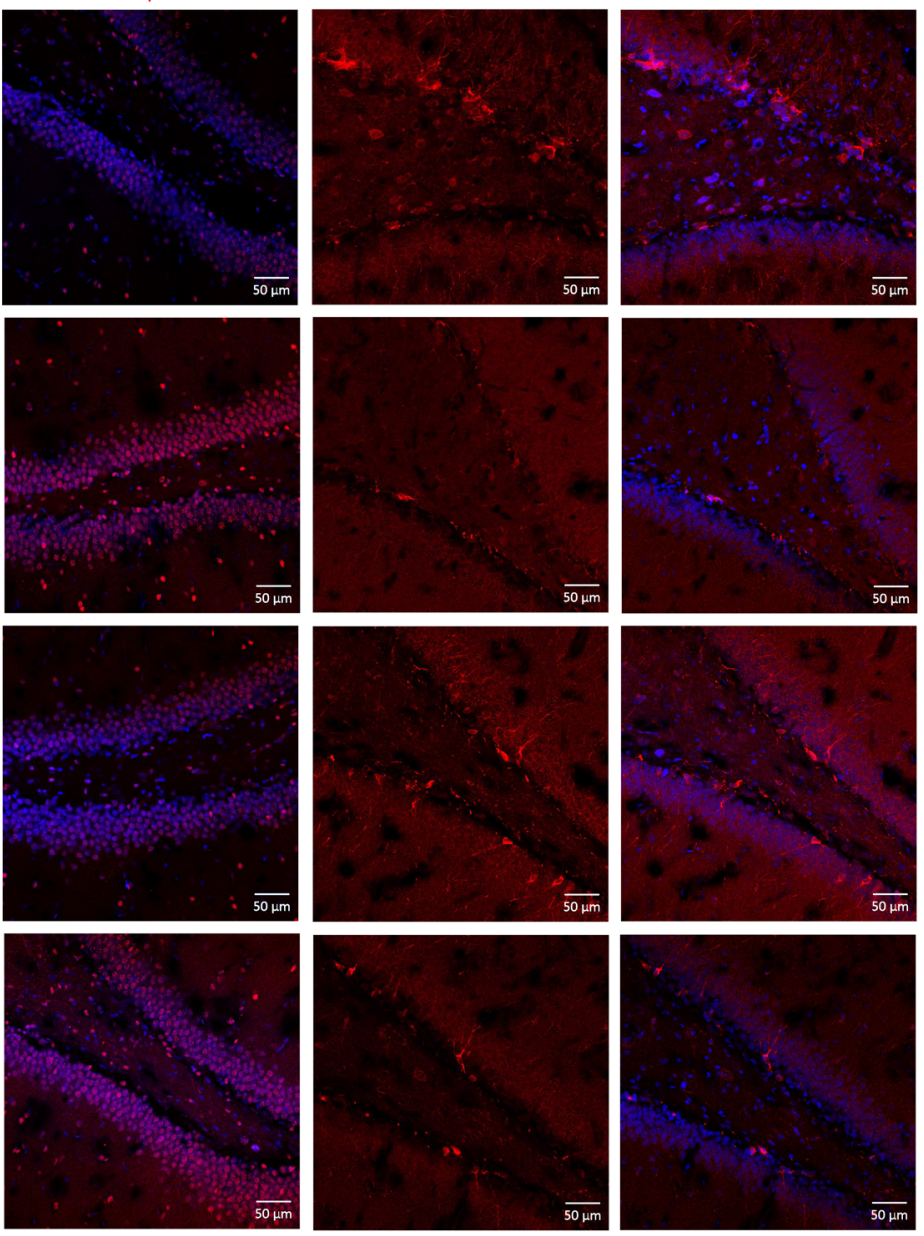

DCX/DAPI
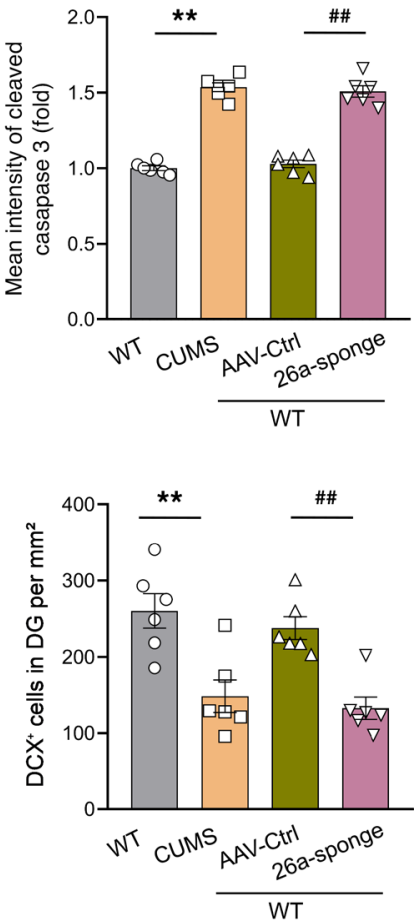

C
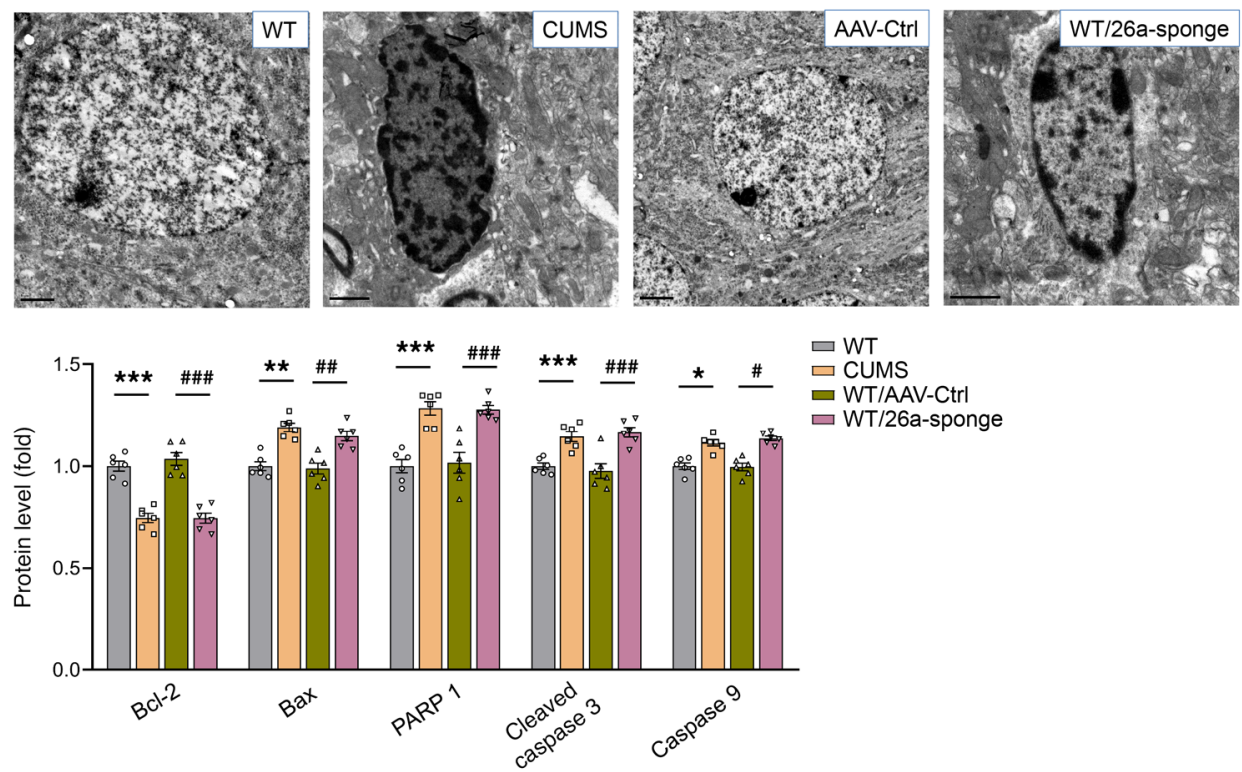

Figure 6. Knockdown of miR-26a-3p within the DG of normal rats induced neuronal apoptosis. (A) Representative confocal microscopic images showing expression of cleaved caspase- 3 and DCX within the DC of different groups. Scale bars: $50 \mu \mathrm{m} . n=6$ rats per group and at least 4-6 images from 1 animal. (B) Knockdown of miR-26a-3p increased protein levels of proapoptotic factors in the DG. $n=6$ rats per group. Western blotting results were from the same samples and run in parallel in different gels. Three independent biological replicate experiments were performed. (C) Representative electron micrographs showing nuclear chromatin abnormalities in DG neurons of different groups. Scale bars: $1 \mu \mathrm{m} . n=6$ per group and at least 5 pyramidal neurons from 1 animal. Immunofluorescence and electron microscopy experiments were repeated at least 3 times and quantitation was done for representative samples from each group. Data are presented as mean \pm SEM. ${ }^{*} P<0.05,{ }^{* *} P<0.01,{ }^{* * *} P<0.001$ vs. WT; ${ }^{\#} P<0.05,{ }^{\# \#} P<0.01,{ }^{\# \#} P<0.001$ vs. AAV-control (WT + AAV-control) by 1-way ANOVA with Tukey's post hoc correction. Ctrl, control. 
A

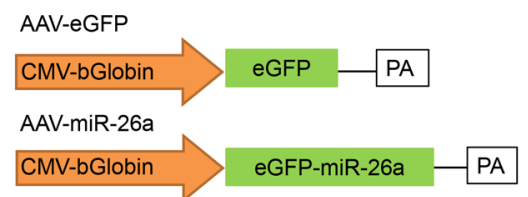

C Viral injection in

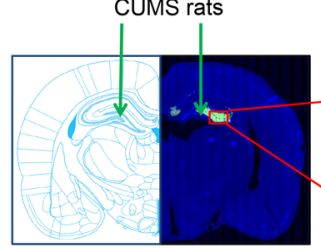

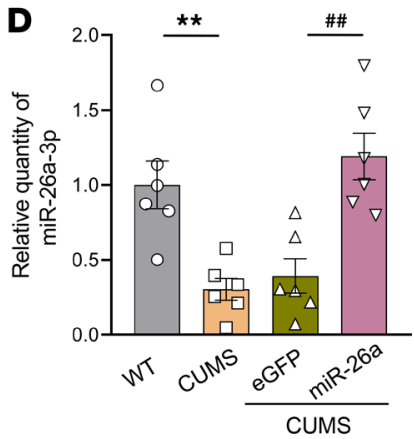

B

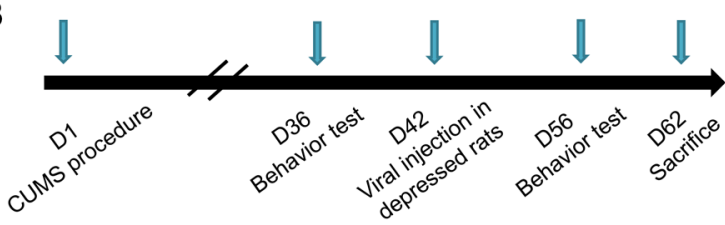

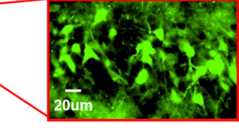

E

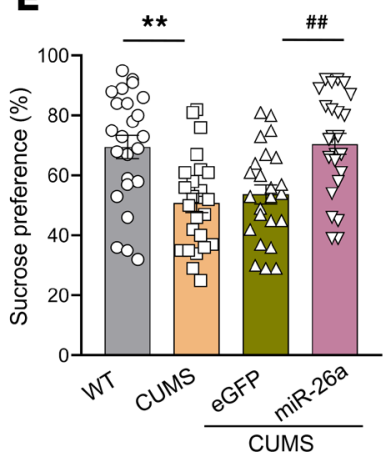

G

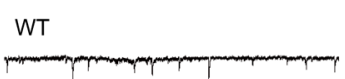

CUMS

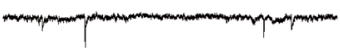

CUMS/eGFP

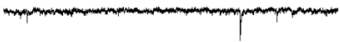

CUMS/miR-26a

$\mathrm{J}_{40 \mathrm{PA}}$
$500 \mathrm{~ms}$
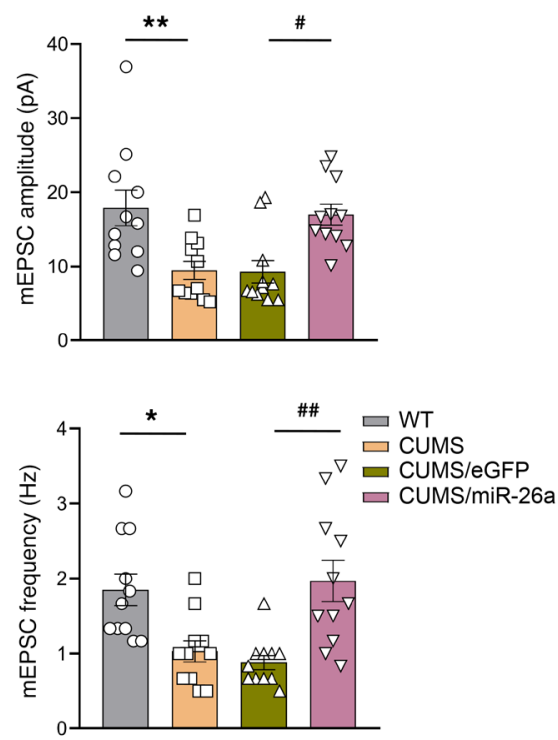

$\mathbf{F}$

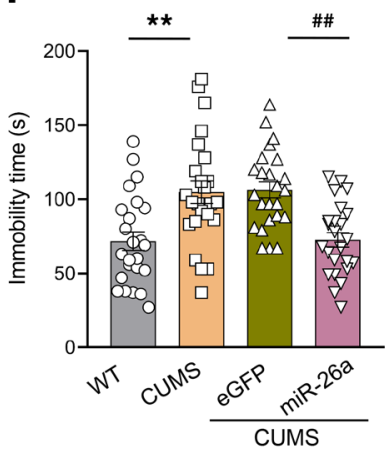

H

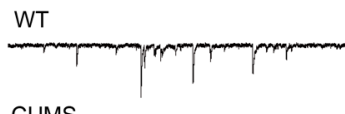

CUMS

CUMS/eGFP

CUMS/miR-26
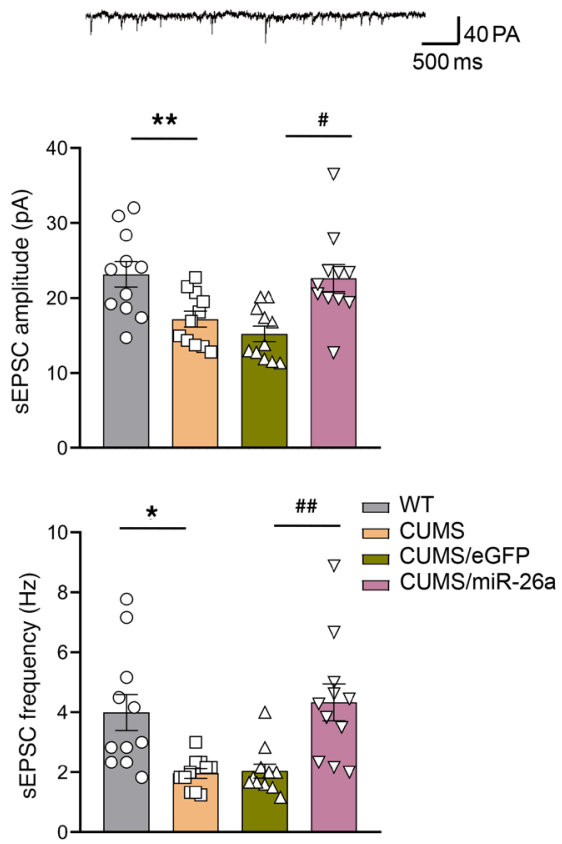

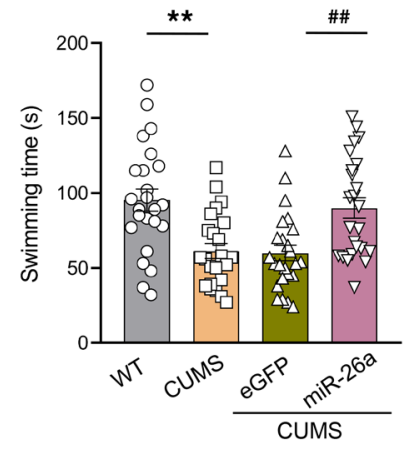

I

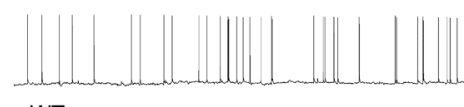

WT
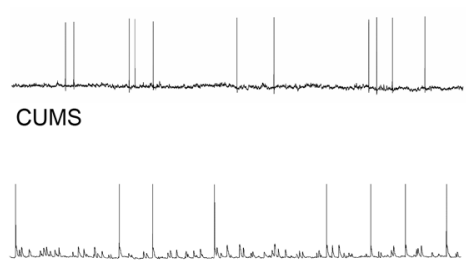

CUMS/eGFP

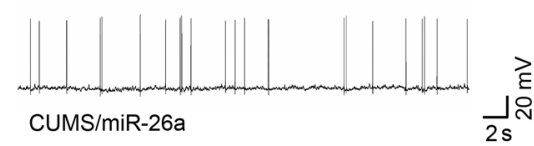

$\square$ WT

$\square$ CUMS

$\square$ CUMS/miR-26a

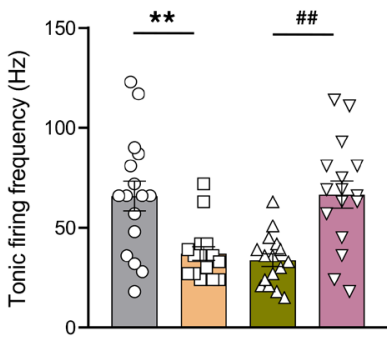


Figure 7. Overexpression of $\mathrm{miR}-26 \mathrm{a}-3 \mathrm{p}$ in the DG of CUMS rats reverses depression-like symptoms produced by CUMS exposure. (A) Schematic of AAV-miR-26a-3p vector used to overexpress miR-26a-3p. (B) Experimental paradigm for CUMS, virus injection, and behavioral testing. (C) Representative site of virus injection in the DG. Scale bar: $20 \mu \mathrm{m}$. (D) Quantitative realtime PCR showing efficiency of miR-26a-3p overexpression in DC regions. $n=6$ rats per group. Three independent biological replicate experiments were performed for each group. (E) Overexpression of miR-26a-3p in the DC of CUMS rats increased sucrose consumption in the sucrose preference test and $(\mathbf{F})$ decreased immobility times and increased swimming times in the forced-swim test. $n=18$ rats per group for behavioral test. Overexpression of miR-26a-3p in DG neurons produced changes in (C) mEPSCs, (H) SEPSCs, and (I) spontaneous burst activity. $n=11$ cells from 6 rats per group in $\mathbf{G}$ and $\mathbf{H} ; n=16$ cells from 6 rats per group in $\mathbf{I}$. Electrophysiological recordings were repeated in at least 3 independent experiments. Data are presented as mean \pm SEM. ${ }^{*} P<0.05$, ${ }^{* *} P<0.01$ vs. WT; ${ }^{\# P}<0.05$, ${ }^{\#} P<$ 0.01 vs. eGFP control (CUMS + AAV-eGFP) by 2-way ANOVA with Tukey's post hoc correction.

nal anomalies in specific brain regions, effective antidepressant therapeutic strategies remain elusive $(25,26)$. Following exposure to CUMS, we found that the resultant depression-like symptoms were accompanied by significant structural and functional neuronal changes within hippocampal DG regions in rats. Previous results from our laboratory have indicated that the depression associated with chronic stress appears to result in neuronal apoptosis and aggravates dendritic spine impairments, while suppression of this neuronal deterioration promotes recovery from depressive behaviors in animal models of depression (27-29). However, details regarding the underlying mechanisms of these pathophysiological processes in depression are not fully understood. Thus, the potential for development of corresponding therapeutic measures targeting these mechanisms remains largely unknown. In this study, we identified differentially expressed miRNAs and assessed their functions with high-throughput sequencing and bioinformatics analysis in an animal model of depression. Notably, miR-26a-3p was found to be significantly downregulated in hippocampal DG tissues of depressed rats, suggesting that this differentially expressed miRNA might play an important role in the development and pathogenesis of depression (30). Previous studies from bioinformatics analysis predicted that miR-26a-3p is involved in numerous pathways, in particular pathways targeting some specific genes associated with the regulation of synaptic plasticity and development within the nervous system (24, $31,32)$. Moreover, as revealed from results of luciferase reporter assays, the present study demonstrated that miR-26a-3p contains conserved seed matches to PTEN mRNA and expression levels of PTEN were decreased with overexpression of miR-26a-3p, while knockdown of miR-26a-3p reversed this reduction in PTEN as demonstrated in vivo. Therefore, these results suggest that miR26a-3p might serve as a regulator in the development of depression. Previous studies have reported that miR-26a-2 possesses antidepressant efficacy by targeting the autoreceptor HTR1A in serotonergic neurons (33). Meanwhile, PTEN was shown to act as a potential target of miR-26a in some cancer research (34-36). However, detailed mechanisms regarding the miR-26a-3p/PTEN dysregulation underlying the neurological damage, in particular whether this neuronal injury is involved in the pathogenesis of depression, are unknown.
Recent studies have demonstrated that the depression associated with chronic stress is accompanied by dendritic remodeling in hippocampal neurons $(37,38)$. Notably, the brain-derived miRNAs could regulate dendritic spine development and may thus be involved in regulating neural plasticity and behaviors $(39,40)$. In order to further explore functions of the miR-26a-3p/PTEN axis as related to depression, we utilized the CUMS-induced rat model of depression. Results showed that chronic stress produced specific changes in synaptic transmission within the hippocampal DG network of rats, consisting of reduced excitatory synaptic transmission as revealed from recordings of both mEPSCs and sEPSCs along with reduced burst activity in DG pyramidal neurons. miR-26a, a brain-enriched miRNA mainly localized within axonal compartments of neurons, has been hypothesized to play a key role in axon development and regeneration $(41,42)$, and is also involved in the formation, maturation, and/or plasticity of synapses via regulating distinct sets of target genes (43). For example, it has been suggested that miR-26a is involved in regulating excitatory neurotransmission under stress conditions (44), and maintains stress resiliency and antidepressant efficacy by targeting the serotonergic autoreceptor HTR1A (33). Therefore, these alterations in synaptic transmission found in the present study may be due to impaired neurobiological functions as correlated with miR-26a-3p deficits induced by stressors. In support of this conjecture, we found that miR-26a-3p can reduce synaptic transmission failure, enhance autophagosomal/lysosomal activities, facilitate synaptic plasticity, and suppress neuronal apoptosis. This capacity for miR-26a-3p to ameliorate synaptic transmission failure may be via an integration of specific neuronal networks participating in the rescue of specific brain dysfunctions related to depression phenotypes. In this way, activation of the miR-26a-3p pathway results in antidepressant effects. Therefore, we hypothesized that neuronal anomalies resulting from chronic stress may be related to synaptic dysfunction in the hippocampal networks eventually leading to a depression-like state in rats. Interestingly, certain stressors not only produce general alterations in neuronal functions but also induce a specific reduction in miR-26a-3p expression, which appears to be a key factor acting on specific neuronal circuits and regulating the activity of DG neurons in depression.

These findings, which show that overexpression of miR-26a$3 p$ improved neurological function recovery along with reductions in depression-like symptoms in CUMS rats, provide persuasive evidence for this hypothesis. Meanwhile, to determine whether miR-26a-3p produces nonspecific responses in the depression-like behavioral alterations, the locomotor activity of rats was measured by the open-field test. The results showed that miR-26a-3p alterations within the DG region have no effect on spontaneous locomotor activity. Moreover, this overexpression of miR-26a$3 \mathrm{p}$ was associated with downregulation of PTEN expression and increases in PI3K and phosphorylated Akt levels. PI3K/Akt and p53 signaling are considered a possible downstream pathway of PTEN, which can then regulate cell functions $(45,46)$. Upregulating PTEN accelerates the inactivation and degradation of phosphorylated PI3K, thereby restraining the PI3K/Akt pathway (47). While we were able to demonstrate the capacity for miR-26a-3p to inhibit the inhibition of PTEN protein translation, the mechanisms underlying the antidepressant-like effects of this pathway 
A

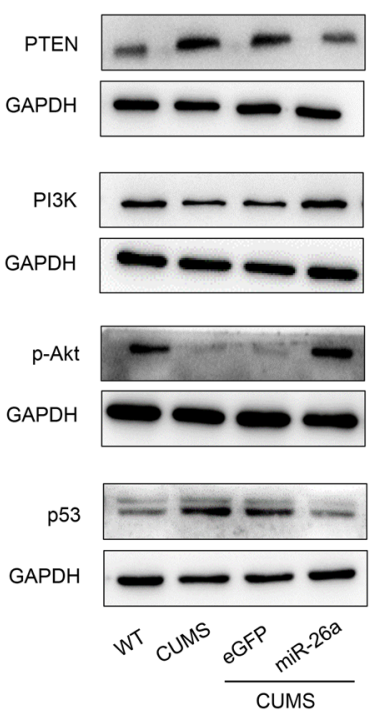

B

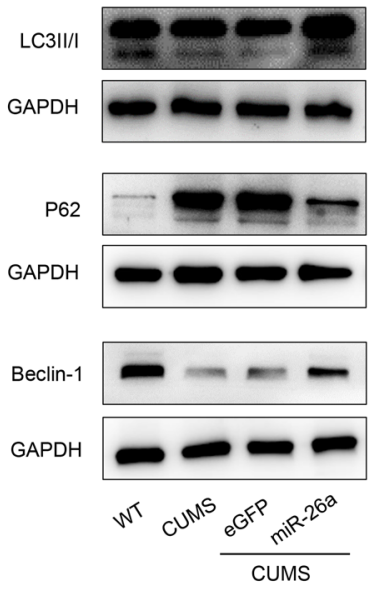

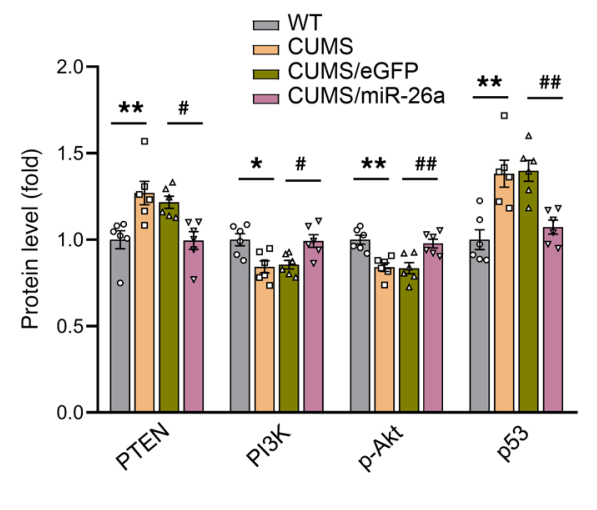

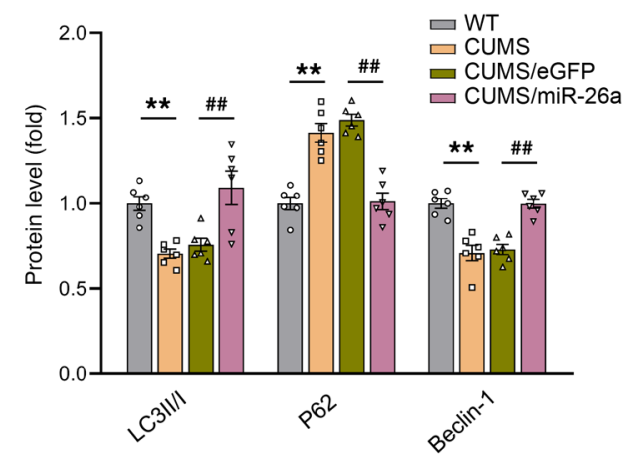

\section{C}
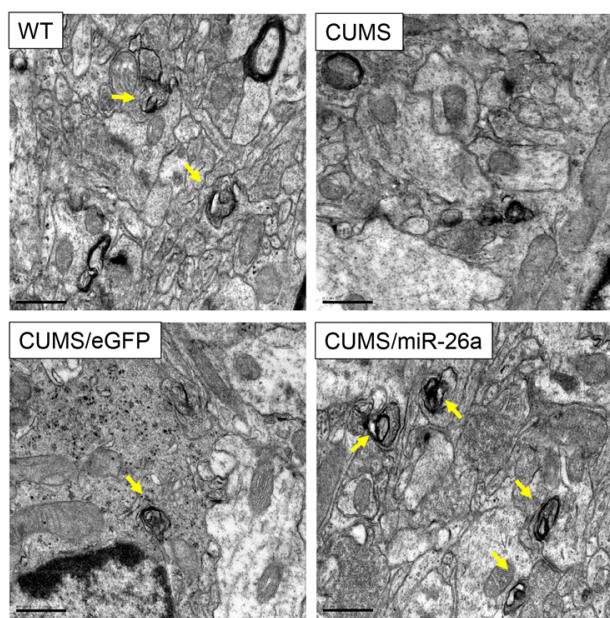

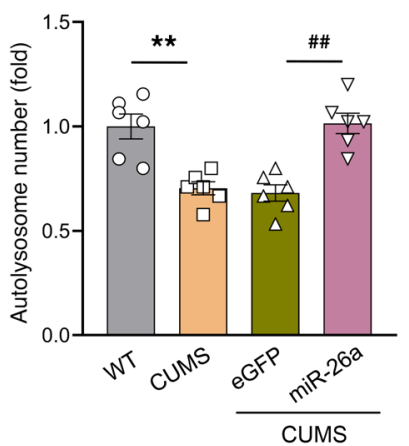

Figure 8. Overexpression of miR-26a-3p in the DG of CUMS rats restored the attenuation in autophagy resulting from CUMS exposure. (A) Overexpression of miR-26a$3 p$ in CUMS rats decreased expression of PTEN and $p 53$ and increased expression of PI3K and phosphorylated Akt within the DG. $n=6$ rats per group. (B) Overexpression of miR-26a-3p increased LC3-II/LC3-I and beclin-1 expression and decreased expression of p62 in CUMS rats. $n=6$ rats per group. (C) Overexpression of miR-26a-3p in the DC of CUMS rats increased the number of autolysosomes. Scale bars: $500 \mathrm{~nm} . n=6$ rats per group with at least 20 micrographs from 1 animal. Experiments were performed in triplicate with 3 biological replicates for all panels. Data are presented as mean \pm SEM. ${ }^{*} P<0.05,{ }^{*} P<0.01$ vs. WT; ${ }^{*} P$ $<0.05,{ }^{\#} P<0.01$ vs. eGFP control (CUMS + AAV-eGFP) by 2-way ANOVA with Tukey's post hoc correction.

remain unclear. Based on the data obtained in the present study, along with data in the literature, we propose that the restoration of synaptic transmission may involve upregulation of brain-derived neurotrophic factor (BDNF) or synaptogenesis (48, 49). In addition, removal of damaged or dysfunctional proteins and organelles via enhancement of autophagosomal/lysosomal activities may, in turn, inhibit apoptotic pathways in neurons (50). Such neuroprotective processes can rebalance functioning of the hippocampal network and subsequently elicit antidepressant effects.

To directly address the issue of whether the PTEN/PI3K/Akt signaling pathway functionally contributes to stress vulnerability as a target of miR-26a-3p, we measured PTEN expression and determined that it was upregulated after knockdown of miR-26a-3p in the DG region. Then, $b p V(p i c)$, an antagonist of PTEN, was used to assess whether miR-26a-3p exerts antidepressant effects via suppression of the PTEN pathway (51-53). bpV(pic) significantly promoted autophagy, reduced apoptotic levels, and restored synaptic transmission and plasticity in hippocampal DG networks lacking miR-26a-3p. Moreover, this inhibition of PTEN also partially alleviated the depression-like behaviors in these CUMS rats. Collectively, these results suggest that the PTEN/PI3K/Akt signaling pathway may act as a downstream component of miR-26a-3p in depression, while the inhibition of this pathway, as accomplished with the PTEN inhibitor bpV(pic), exerts a neuroprotective effect in depressed rats. It should be noted that the present study mainly investigated the neuroprotective effects of the PTEN inhibitor $\mathrm{bpV}$ (pic) in the central nervous system. However, PTEN also exerts multiple functions in many diseases besides the nervous system via complicated molecular mechanisms. For example, PTEN acts as a tumor suppressor in breast cancer (54), glioma (55), and leukemia (56). Therefore, the potential side effects or off-target effects in animals 
A

Syn
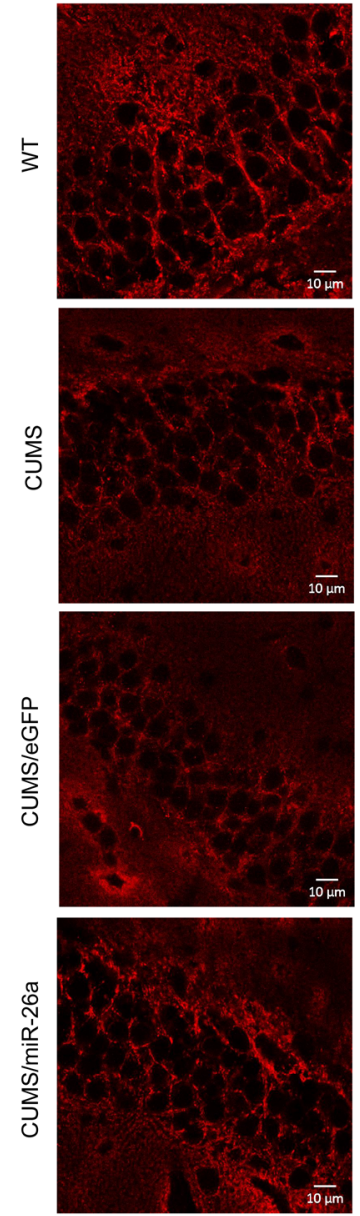

B
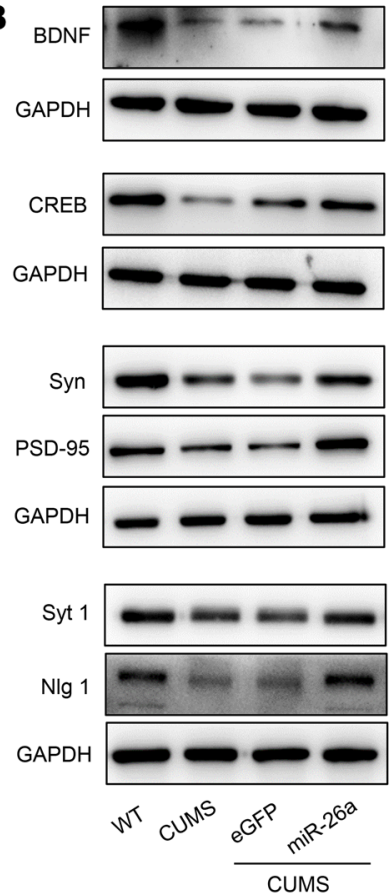

Syn/DAPI
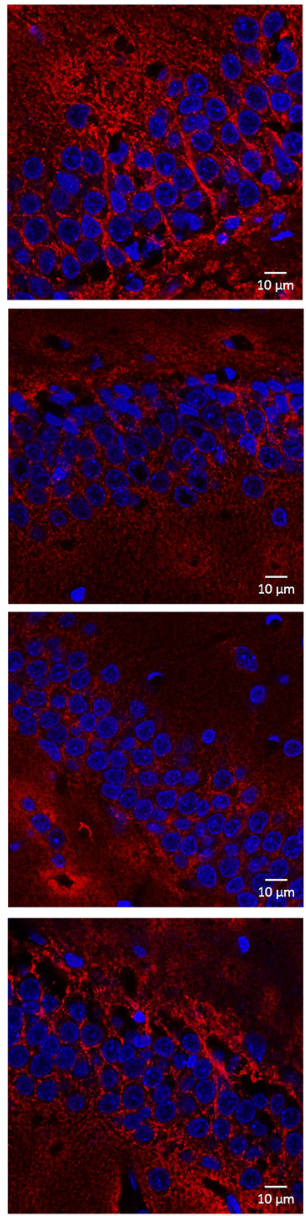

C

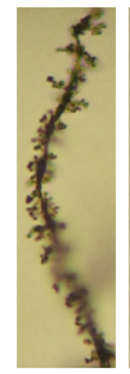

w
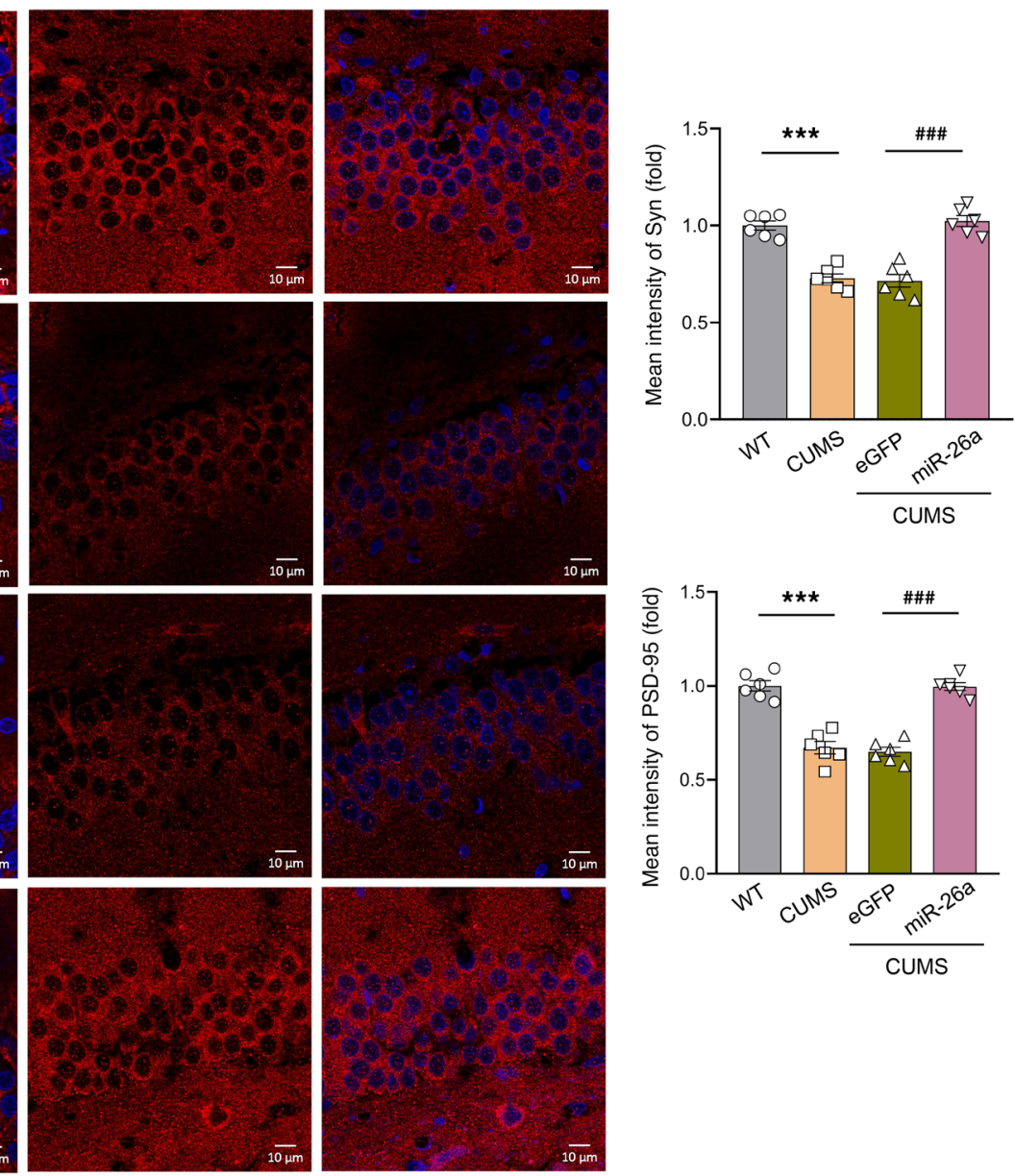

PSD-95/DAPI
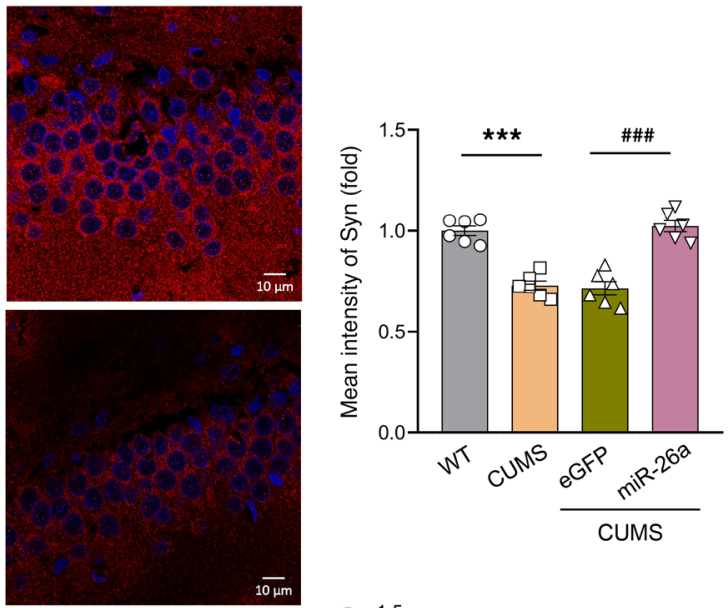

$10 \mu \mathrm{m}$
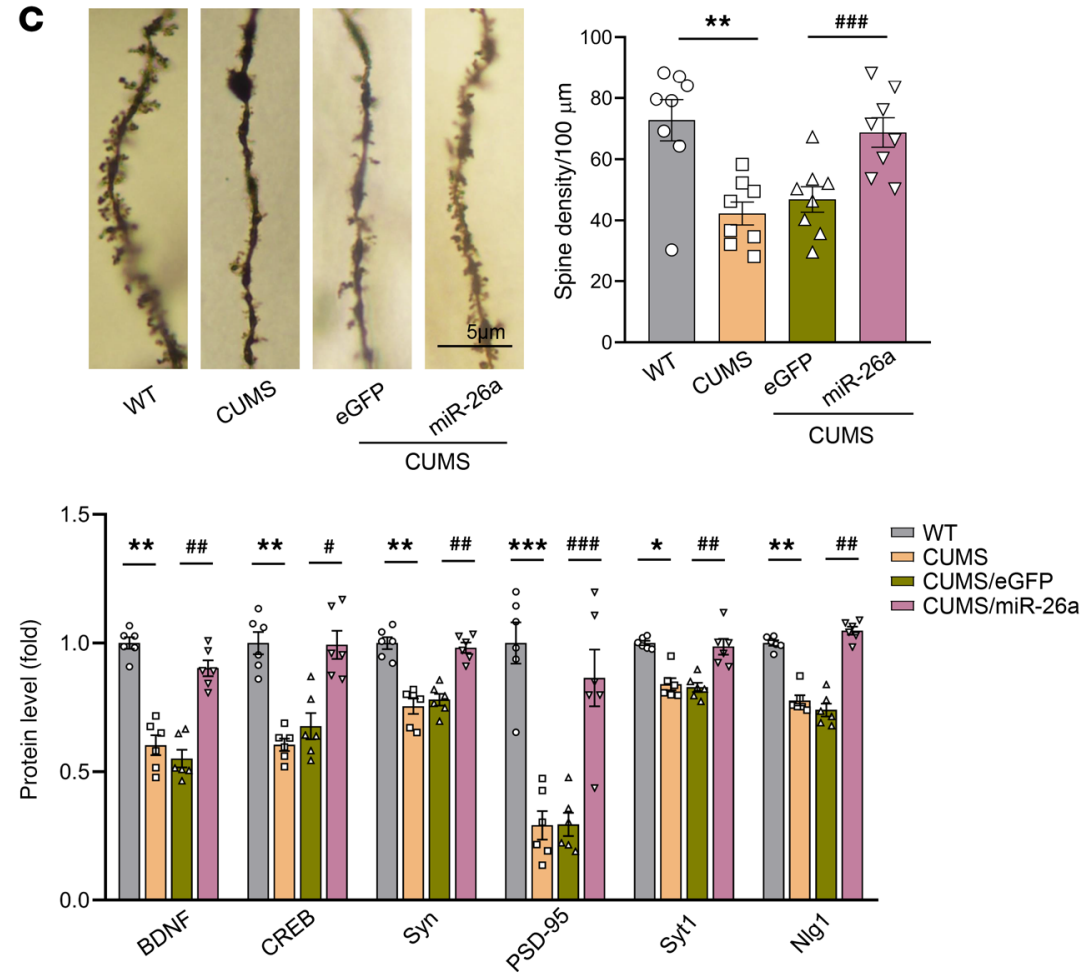
Figure 9. Overexpression of $\mathrm{miR}-26 \mathrm{a}-3 \mathrm{p}$ in the DG of CUMS rats restored the dysregulation of neuroplasticity resulting from CUMS exposure. (A) Representative confocal microscopic images showing the expression levels of Syn and PSD-95 within the DC. Scale bars: $10 \mu \mathrm{m} . n=6$ rats per group and at least 4-6 images from 1 animal. (B) Overexpression of miR-26a-3p increased protein levels of neuroplasticity-related mediators in CUMS rats. $n=6$ rats per group. Western blotting results were from the same samples and run in parallel in different gels. Independent biological replicate experiments were repeated 3 times. (C) Representative images and summary of data showing dendritic spines in DG neurons. Scale bar: $5 \mu \mathrm{m} . n=8$ rats per group and at least 5 pyramidal neurons from 1 animal. Immunofluorescence and Golgi staining were repeated at least 3 times and quantitation was done for representative samples from each group. Data are presented as mean \pm SEM. ${ }^{*} P<0.05,{ }^{* *} P<0.01,{ }^{* * *} P<0.001$ vs. WT; ${ }^{*} P<0.05$, ${ }^{\# \# P}$ $<0.01$, \#\#\# $P<0.001$ vs. eGFP control (CUMS + AAV-eGFP) by 2 -way ANOVA with Tukey's post hoc correction. Syt1, synaptotagmin 1; NIg1, neuroligin 1.

using this peripheral administration of $\mathrm{bpV}(\mathrm{pic})$ should be taken into consideration, and determining the potential effects of PTEN inhibition on depression will require further investigation.

In conclusion, the findings of this report lead to the hypothesis that miR-26a-3p, via suppression of the PTEN/PI3K/Akt pathway, plays an important role in the alleviation of neuronal anomalies coupled with depression as induced by CUMS. Accordingly, these results have important biological and clinical significance with regard to the potential identification and treatment of depressive disorders.

\section{Methods}

Animals and housing conditions. Male Wistar rats (180-200 g body weight) were obtained from the Shandong University Animal Centre. All efforts were made to minimize the number and suffering of the animals used in the experiments. Rats were housed under standard laboratory conditions for at least 1 week prior to experimental procedures.

CUMS model. The rat model of depression consisted of the CUMS procedure as described previously with minor modifications (57). Briefly, rats were housed individually and subjected to chronic stressors including clipping tails (1 minute), physical restraint (2 hours), 5 -minute cold swimming $\left(4^{\circ} \mathrm{C}\right)$, overnight illumination, cage shaking (2 hours), 24-hour food and water deprivation, foot shock (0.5 mA, 0.5 seconds), and wet bedding ( 24 hours). One stressor was applied daily to each rat in a random order over a 5 -week period.

Behavioral tests. The following behavioral tests were used to assess depression-like behaviors in rats after the 5 weeks of CUMS exposure.

Sucrose preference test. The sucrose preference test was conducted as described previously (57). In the adaptation phase, rats were permitted accessed to 2 bottles, each containing $1 \%$ sucrose solution for 24 hours, and then 1 bottle was replaced with one containing tap water for the subsequent 24 -hour period. In the test phase, after 24 hours of food and water deprivation, rats were permitted free access to 2 bottles for 3 hours, one containing $100 \mathrm{~mL}$ of sucrose solution $(1 \%, w / v)$ and the other $100 \mathrm{~mL}$ of tap water. Sucrose preference was calculated as sucrose consumption/ (water consumption + sucrose consumption) $\times 100$.

Forced-swim test. The forced-swim test was conducted according to procedures described in previous studies $(58,59)$. On the training day, rats were placed individually in a water-containing cylinder (height, $80 \mathrm{~cm}$; diameter, $30 \mathrm{~cm}$; temperature, $25^{\circ} \mathrm{C}$ ) for
15 minutes of forced swimming. After 24 hours, each rat was placed in the cylinder for a 5-minute test period. Durations of immobility (floating except for movements required to maintain the head above water) and swimming were recorded by an experimenter blinded to the treatment group.

miRNA library construction and sequencing. Total RNA isolated from hippocampal DG tissue obtained from control and CUMS groups was used for miRNA library preparation and sequencing, as well as for the subsequent qPCR verification. Briefly, total RNA samples were fractionated in a $15 \%$ Tris-borate-EDTA (TBE) polyacrylamide gel (Invitrogen) and small RNAs ranging from 18 to 30 nucleotides were purified and used for library preparation. Small RNAs were reverse transcribed into cRNA and amplified by PCR. The PCR products were sequenced using the Illumina HiSeq 2500 platform. The follow-up qPCR analyses were performed to validate the expression levels of some miRNAs from the microarray analysis. The miRNA pathway prediction was performed based on DIANA-miRPath (24). The Database for Annotation, Visualization and Integrated Discovery (DAVID) functional annotation was performed for target genes (31). miRNA target genes implicated in the pathway were investigated by using the KEGG database (32). A $P$ value of less than 0.05 was used as the criterion for statistical significance. The miRNA library preparation and sequencing were performed by RiboBio.

Dual-luciferase reporter assay. Potential targets of miR-26a-3p and PTEN were predicted by TargetScan (www.targetscan.org) and miRDB (www.mirdb.org). For the in vitro luciferase assay, $100 \mathrm{ng}$ of luciferase reporter plasmid (pmirGLO-PTEN or pmirGLO-PTENMUT recombinant vector) was cotransfected with $400 \mathrm{ng}$ of miR26a-3p mimic (overexpression sequence of miR-26a-3p) or miR-NC into cultured HEK293T cells using Lipofectamine 2000 (Invitrogen, 11668027). Twenty-four hours after transfection, luciferase activity was detected in HEK293T cells with the Dual-Luciferase Reporter Assay System (Promega; ref. 60).

Stereotaxic injection of the AAV vector. The AAV9-CMV-eGFPmiR-26a-3p vector (AAV-miR-26a-3p) was constructed to overexpress miR-26a-3p. The following primer sequence was used for expressing fragments of miR-26a-3p: ACGAGCTGTACAAGGCTAGCTAACCCCTTCTCTTTGACAGTAG (Gene Chem Co). A sponge sequence form of miR-26a-3p was expressed in the AAV to produce the AAV9CMV-eGFP-Sponge(miR-26a-3p)-WPRE vector (AAV-miR-26a-3psponge), which acts as a sponge and inhibits the functions of endogenous miR-26a-3p. The inverse complementary sequence of mature miR-26a-3p is GTGCAAGTAACCAAGAATAGG (Gene Chem Co). For viral injection, rats were anesthetized with sodium pentobarbital (150 mg/kg, i.p.) and placed in a stereotaxic frame (Stoelting). The skin between the 2 ears of rats was shaved with a trimming machine and then sanitized with Betadine. We used scalpel to make an anterior-posterior incision of approximately $1.5 \mathrm{~cm}$ in length between the ears. The surface of the skull was cleaned with a cotton swab until the bregma, which is located at the intersection between the coronal suture and sagittal sutures, was visible. Using the tip of the microliter syringe to point to the bregma point, the coordinates of these 3 axes were considered the zero point. According to coordinates of the hippocampal regions in the Rat Brain Atlas, the virus injection site was focused on the center of hippocampus according to the following coordinates relative to the bregma, in $\mathrm{mm}:-3.24$ at the anterior/posterior axis, \pm 0.5 at the lateral/medial axis, and -4.8 at the dorsal/ventral axis. 
A

DCX

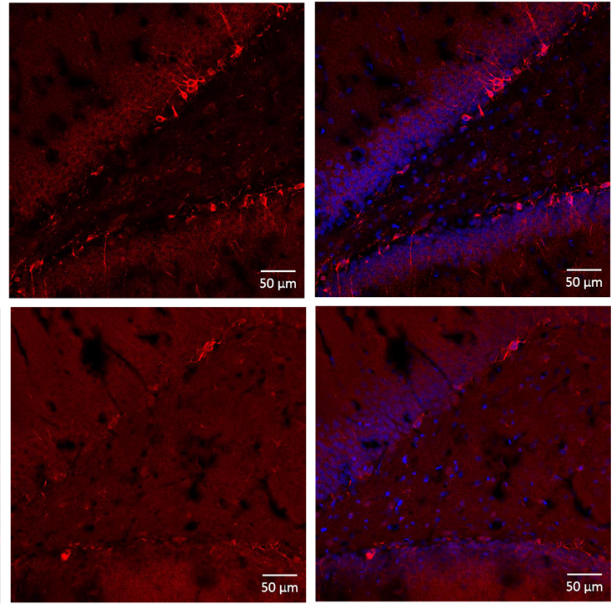

5

$\sum_{0}^{\infty}$

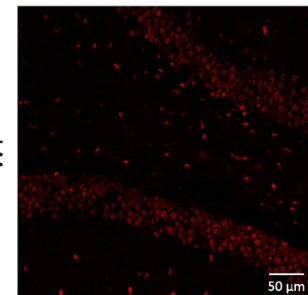

$\overline{50 \mathrm{~mm}}$
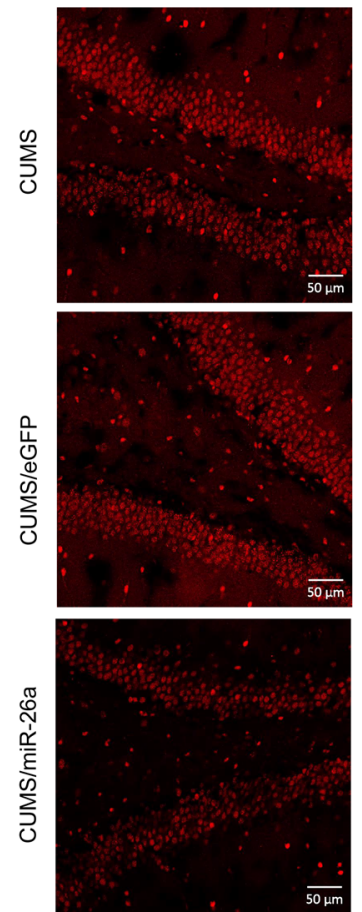

B
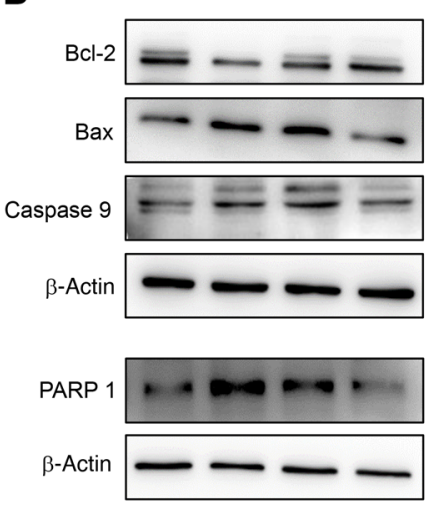

Cleaved caspase 3

$\beta$-Actin
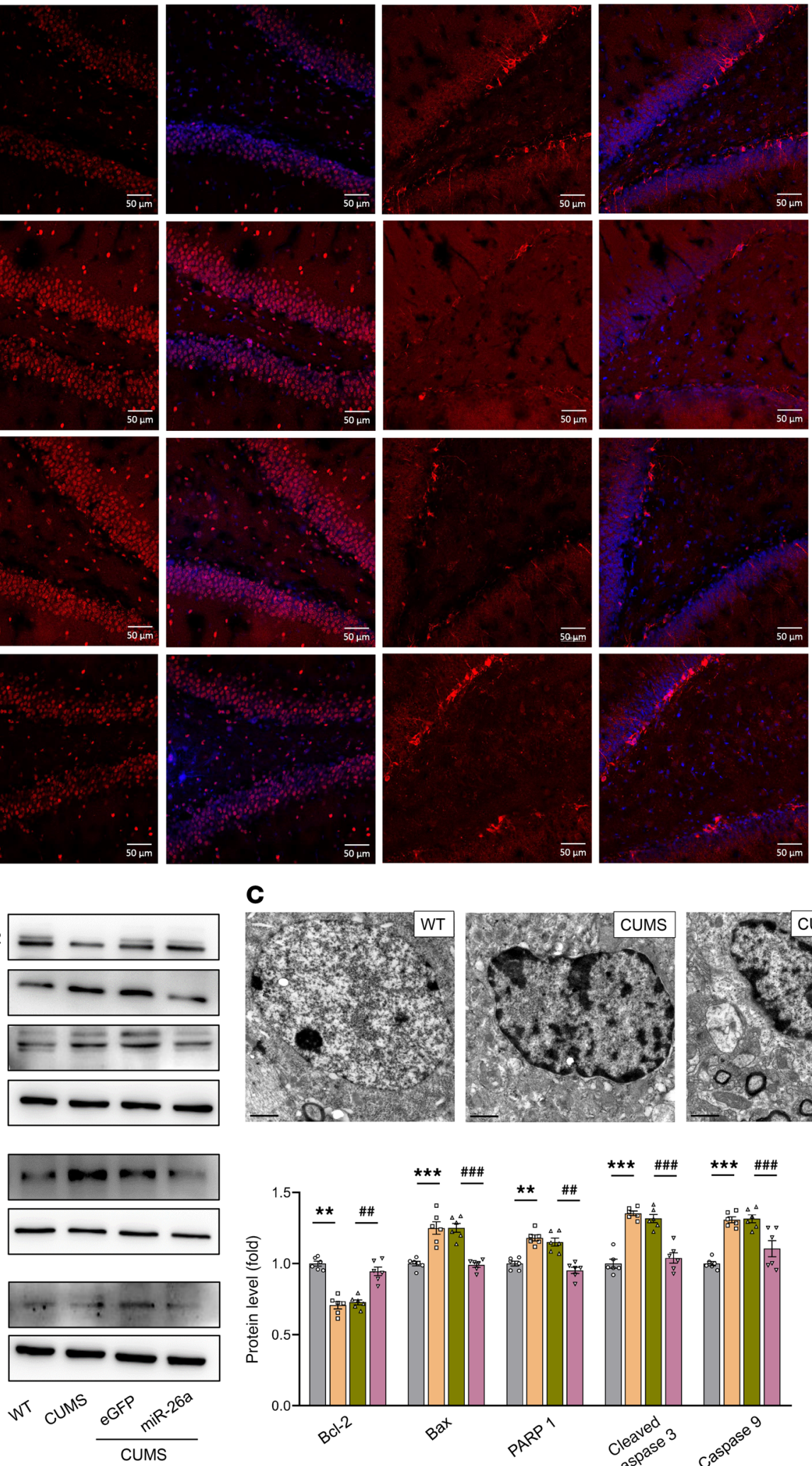

\section{C}

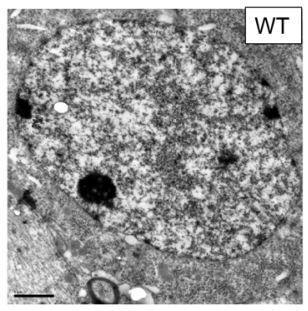

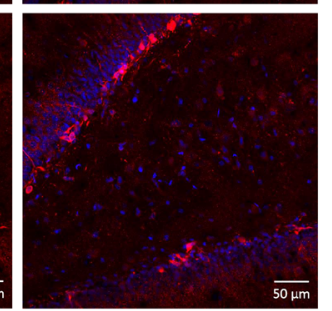
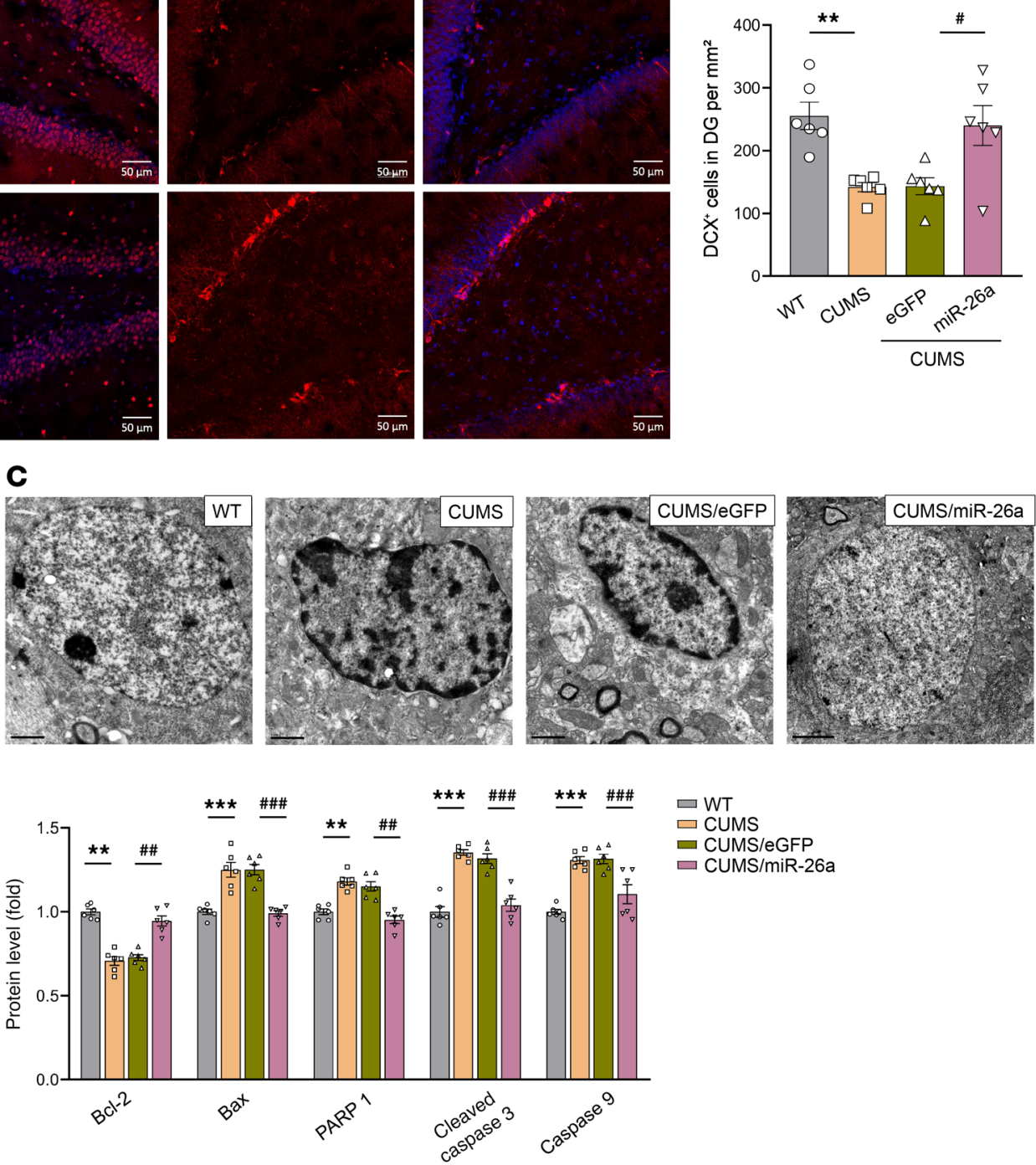
Figure 10. Overexpression of miR-26a-3p within the DG of CUMS rats suppressed neuronal apoptosis resulting from CUMS exposure. (A) Representative confocal microscopic images showing expression of cleaved caspase- 3 and DCX within the DG. Scale bars: $50 \mu \mathrm{m} . n=6$ rats per group and at least 4-6 images from 1 animal. (B) Overexpression of miR-26a-3p decreased protein levels of proapoptotic factors in CUMS rats. $n=6$ rats per group. Western blotting results of $\mathrm{Bcl}-2$, Bax, and caspase- 9 were from the same samples and run in parallel in different gels. Independent biological replicate experiments were repeated 3 times. (C) Representative electron micrographs showing nuclear chromatin abnormalities in DG neurons. Scale bars: $1 \mu \mathrm{m} . n=4$ rats per group and at least 20 micrographs from 1 animal. Immunofluorescence and electron microscopy experiments were repeated at least 3 times and quantitation was done for representative samples from each group. Data are presented as mean \pm SEM. ${ }^{* *} P<0.01$ ${ }^{* * *} P<0.001$ vs. WT; ${ }^{\#} P<0.05,{ }^{\# \# P}<0.01,{ }^{\# \# \# P} P 0.001$ vs. eGFP control (CUMS + AAV-eGFP) by 2-way ANOVA with Tukey's post hoc correction.

Shallow holes were drilled only in the skull bone with a fine drill bit. A microliter syringe was placed above the hole and slowly dropped vertically until it reached the target hippocampal regions. Purified AAV $\left(\sim 1 \times 10^{12}\right.$ infection units per $\left.\mathrm{mL}, 1-1.5 \mu \mathrm{L}\right)$ was infused bilaterally into hippocampal regions at a rate of $150 \mathrm{~nL} / \mathrm{min}$. The microliter syringe remained in the injection site for at least 5 minutes after infusion and was then slowly withdrawn. Infection efficiency assays were performed a minimum of 14 days after virus injection. Injection sites were verified and only data from rats with correct injection sites were included in the analyses.

Experimental design. Rats were randomly assigned to one of the following groups: (a) WT controls (nonstressed and noninjected group), (b) WT + AAV-control (WT/eGFP construct), (c) WT + AAVmiR-26a-3p-sponge, (d) CUMS (5-week chronic stress), (e) CUMS + AAV-control (CUMS/eGFP construct), (f) CUMS + AAV-miR-26a$3 \mathrm{p}, \quad(\mathrm{g}) \mathrm{bpV}(\mathrm{pic})+\mathrm{WT} / \mathrm{AAV}-\mathrm{miR}-26 \mathrm{a}-3 \mathrm{p}$-sponge, and (h) DMSO + WT/AAV-miR-26a-3p-sponge. For bpV(pic) (catalog AG-CR10043-M005, Adipogen) treatment, rats were given bpV(pic) $(0.2 \mathrm{mg} /$ $\mathrm{kg}$, i.p.) 4 times at an interval of 3 hours as previously described (61), and AAV-miR-26a-3p-sponge was injected 30 minutes after the last injection. bpV(pic) was dissolved in DMSO (MilliporeSigma) and the concentration of DMSO did not exceed $0.1 \%$ of the total volume.

Hippocampal slice preparations and whole-cell recordings. Rats were anesthetized using pentobarbital and rapidly decapitated. Hippocampal coronal slices (300 $\mathrm{mm}$ in thickness) were sectioned with a vibratome (VT-1200s, Leica) in oxygenated (95\% $\left.\mathrm{O}_{2} / 5 \% \mathrm{CO}_{2}\right)$, ice-cold cutting solution ( $\mathrm{pH} 7.4$ ) containing (in $\mathrm{mM}$ ) 30 glucose, 2.5 $\mathrm{KCl}, 26 \mathrm{NaHCO}_{3}, 7 \mathrm{MgSO}_{4}, 1 \mathrm{NaH}_{2} \mathrm{PO}_{4}, 1 \mathrm{CaCl}_{2}, 119$ choline chloride, 1 kynurenic acid, 3 sodium pyruvate, and 1.3 sodium L-ascorbate. Slices were quickly transferred to a recovery solution containing (in mM) $85 \mathrm{NaCl}, 2.5 \mathrm{KCl}, 1.25 \mathrm{NaH}_{2} \mathrm{PO}_{4}, 0.5 \mathrm{CaCl}_{2}, 4 \mathrm{MgCl}_{2}, 24 \mathrm{NaH}-$ $\mathrm{CO}_{3}, 25$ glucose, and 50 sucrose. The slices were allowed to recover for 30 minutes at $36^{\circ} \mathrm{C}$ and then a minimum of 1 hour at room temperature before recording. Whole-cell patch-clamp recordings in voltage-clamp mode were performed on neurons within the DG hippocampus. In the process of voltage-clamp recordings, patch pipettes (3-5 M $\Omega$ ) were filled with a solution ( $\mathrm{pH}$ 7.3) containing (in mM) $125 \mathrm{CsCl}_{2}, 5 \mathrm{NaCl}, 4$ Hepes, 0.2 EGTA, 0.2 NaGTP, $2 \mathrm{MgATP}$, 7 phosphocreatine, and $2 \mathrm{MgCl}_{2}$. EPSCs were detected at a holding potential of $-70 \mathrm{mV}$ with $50 \mathrm{mM} \mathrm{AP-5}$ (Sigma-Aldrich; catalog A5282) and $50 \mathrm{mM}$ picrotoxin (R\&D Systems, catalog 1128) pres- ent in the artificial cerebrospinal fluid (ACSF) used for perfusion. mEPSCs were recorded with the application of $1 \mathrm{mM}$ tetrodotoxin (R\&D Systems; catalog 1078/1) in external solutions. During recordings, slices were continuously perfused with ACSF at a rate of approximately $2 \mathrm{~mL} / \mathrm{min}$ and at $32^{\circ} \mathrm{C} \pm 1^{\circ} \mathrm{C}$. sEPSCs and mEPSCs were analyzed using the Mini Analysis Program (Synaptosoft). Event counts were performed by an experimenter blinded to the group identity. All recording data were filtered at $2 \mathrm{kHz}$ and digitized at $10 \mathrm{kHz}$. Data were acquired using a Digidata $1440 \mathrm{~A}$ and pCLAMP 10.6 software (Molecular Devices). Only neurons with sufficiently negative resting membrane potentials $(\leq-65 \mathrm{mV})$ and an absence of spontaneous firing were included in the analysis.

Immunofluorescence assay. One day after behavioral tests, rats were anesthetized and perfused with $4 \%$ paraformaldehyde (PFA). Brains were postfixed in PFA overnight at $4^{\circ} \mathrm{C}$ followed by a graded dehydration and then cut into serial coronal frozen slices $(30 \mu \mathrm{m})$. Slices were incubated with primary antibodies consisting of anti-Syn (1:50, cata$\log$ 9020), anti-MAP-2 (1:100, catalog 4542), anti-cleaved caspase-3 (1:100, catalog 9661), anti-DCX (1:200, catalog 4604) (all Cell Signaling Technologies); anti-PSD-95 (1:100, catalog 20665-1-AP, Proteintech Group); and anti-nestin (1:50, catalog PA5-47378, Thermo Fisher Scientific) followed by a fluorophore-conjugated secondary antibody (1:200, catalog SA00013-1, Proteintech Group). Slices were washed in PBS and counterstained with 4',6-diamidino-2-phenylindole dihydrochloride (DAPI) (catalog C0060, Solarbio) for 7 minutes. Images were captured with a laser scanning confocal microscope (LSM780, Carl Zeiss). At least 4 to 6 images were obtained for each rat for analysis by Image-Pro Plus 6.0 software (Media Cybernetics). To obtain the mean intensity of immunofluorescence, this software was used to circle the hippocampal DG area to measure the area size and the total intensity of immunofluorescence on the area. To obtain the number of positive cells in the DG per $\mathrm{mm}^{2}$, the software was used to circle the hippocampal DG area to measure the size of the area and the total number of positive cells.

Golgi staining. Golgi staining was performed to examine changes in neuronal dendritic spines using the FD Rapid GolgiStain Kit (PK401, FD Neuro-Technologies, MD21041) according to the manufacturer's instructions. Briefly, rats were anesthetized and brains rapidly removed and immersed in an impregnation solution $(\mathrm{A} / \mathrm{B}=$ $1: 1$, total $15 \mathrm{~mL} /$ rat) for 2 weeks. Brain samples were cut into $100 \mu \mathrm{m}$ coronal slides and cleaned in xylene. Slides were then coverslipped with rhamsan gum for light microscopic observation. Apical dendrites of neurons were chosen for morphological analysis. For each group, at least 4 to 6 dendritic segments per neuron were randomly selected and at least 5 pyramidal neurons were analyzed per rat. The number of spines was analyzed with Image-Pro Plus 6.0 software.

Electron microscopic analysis. Transmission electron microscopy was used to examine synapses and ultrastructures of neurons. DG tissue samples $(1 \times 1 \times 1 \mathrm{~mm})$ were carefully dissected and placed in $2.5 \%$ glutaraldehyde at $4^{\circ} \mathrm{C}$ for 2 to 4 hours. The tissue was fixed with $1 \% \mathrm{OsO}_{4}$ in $0.1 \mathrm{M} \mathrm{PBS}$ ( $\mathrm{pH}$ 7.4) for 1 hour and subjected to a graded ethanol dehydration, followed by infiltration with acetone, a mixture of 1:1 epon-acetone, and $100 \%$ epon in sequence overnight. Tissues were embedded in resin, cut into ultrathin sections ( $70 \mathrm{~nm}$ thick), and stained with $4 \%$ uranyl acetate for 20 minutes followed by staining with $0.5 \%$ lead citrate for 15 minutes. Sections were examined under a transmission electron microscope (Philips Tecnai 20 U-Twin). At least 
A

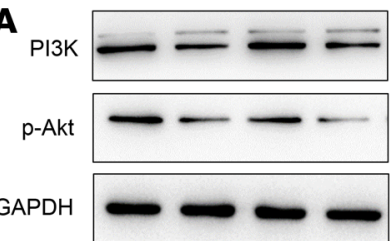

p53

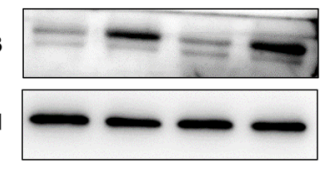

WT $\frac{W^{T} B^{2 N} D^{N S O}}{\text { AAV-26a-sponge }}$

$\square$ WT

$\square$ 26a-sponge

BPV/26a-sponge

$\square$ DMSO/26a-sponge

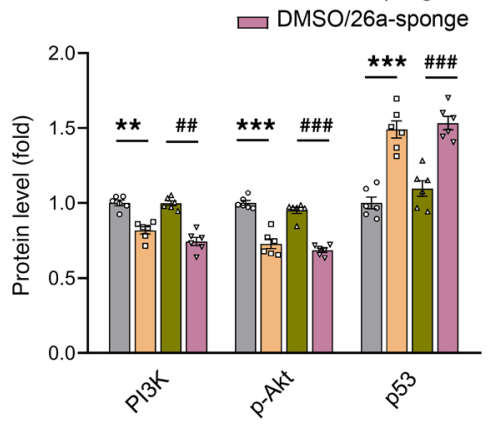

D

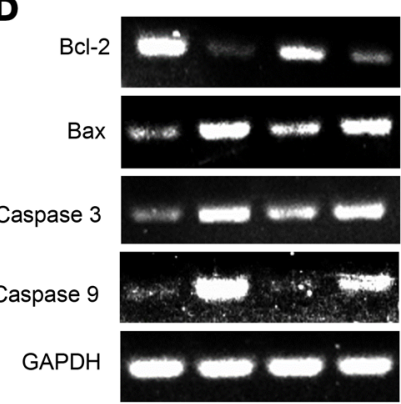

$W^{N} \frac{W^{N} B^{P V} a^{N S^{O}}}{\text { AAV-26a-sponge }}$

$\square \mathrm{WT}$

$\square$ 26a-sponge

$\square$ BPV/26a-sponge

$\square$ DMSO/26a-sponge

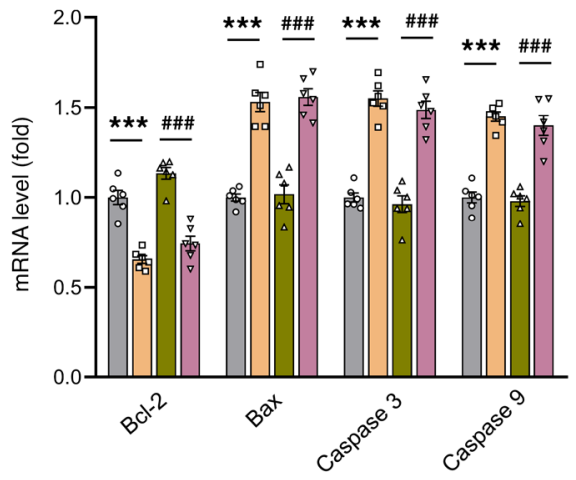

B
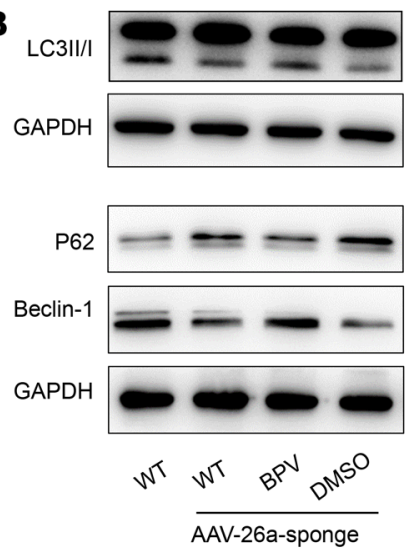

$\square$ WT

$\square$ 26a-sponge

BPV/26a-sponge

$\square$ DMSO/26a-sponge

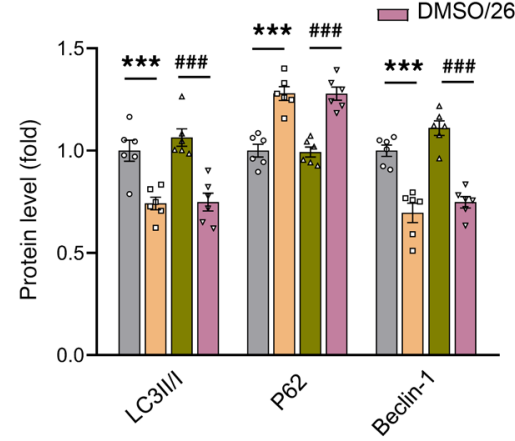

$\mathbf{E}$

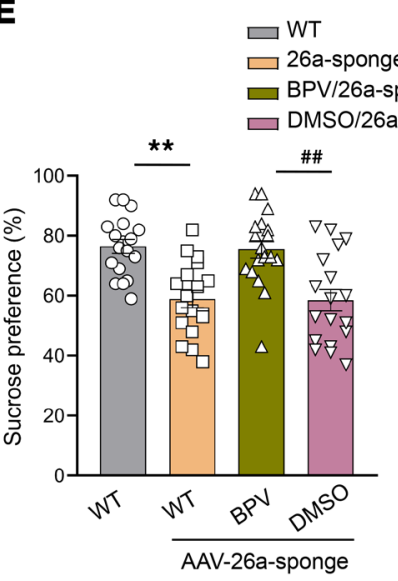

$\mathbf{F}$

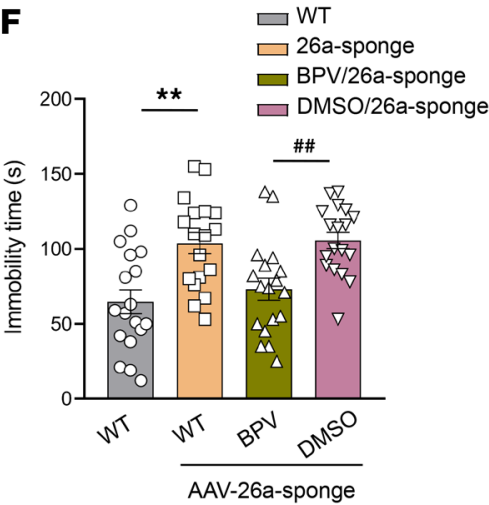

C
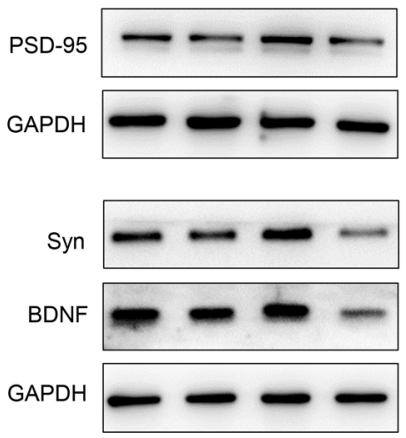

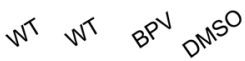

AAV-26a-sponge

$\square$ WT

$\square$ 26a-sponge

$\square$ BPV/26a-sponge

$\square$ DMSO/26a-sponge

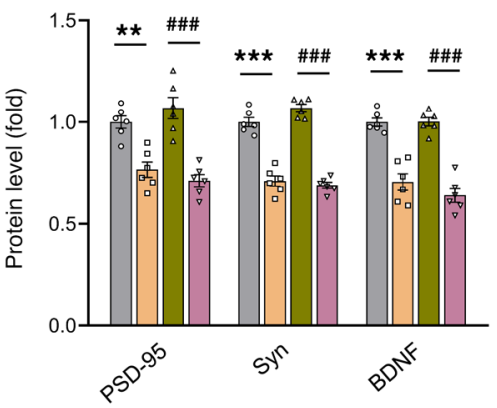

G
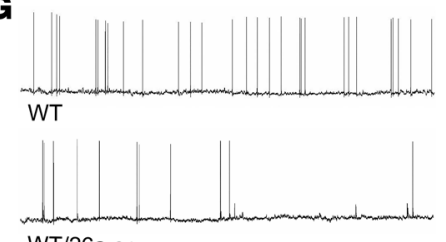

WT/26a-sponge

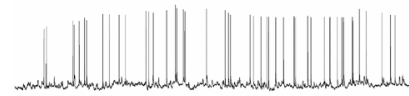

WT/26a-sponge+BPV
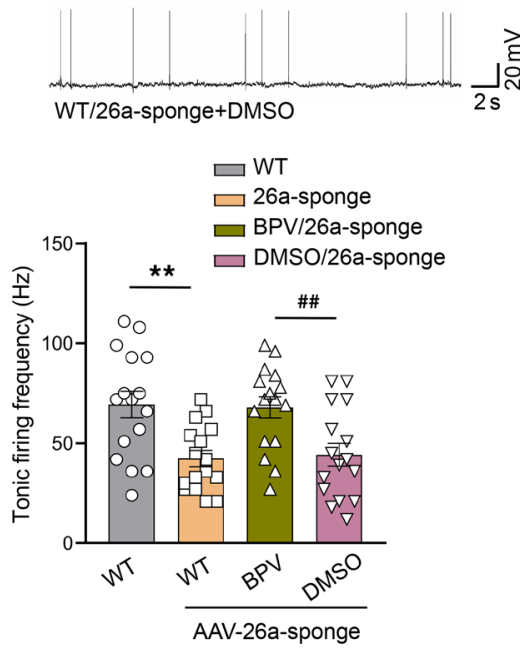
Figure 11. PTEN inhibition attenuated neuronal and behavioral anomalies resulting from miR-26a-3p deficits in the DC. (A) bpV(pic) treatment increased expression of PI3K and phosphorylated Akt and decreased $p 53$ expression levels in miR-26a-3p-knockdown rats. Western blotting results of $\mathrm{PI} 3 \mathrm{~K}$ and $\mathrm{p}$-Akt were from the same samples and run in parallel in different gels. $n=6$ rats per group with 3 independent biological replicate experiments. (B) bpV(pic) treatment increased LC3-II/LC3-I and beclin-1 expression, and decreased expression of p62 in miR-26a-3p-knockdown rats. Western blotting results for $\mathrm{p} 62$ and beclin-1 were from the same samples and run in parallel in different gels. $n=6$ rats per group with 3 independent biological replicate experiments. (C) bpV(pic) treatment increased protein levels of BDNF, PSD-95, and Syn within the DG of miR-26a-3p-knockdown rats. Western blotting results for Syn and BDNF were from the same samples and run in parallel in different gels. $n=6$ per rats group with 3 independent biological replicate experiments. (D) bpV(pic) treatment decreased mRNA levels of Bax, caspase-3, and caspase-9, and increased Bcl-2 mRNA levels in miR$26 a-3 p-k n o c k d o w n$ rats. $n=6$ rats per group with 3 independent biological replicate experiments. (E) bpV(pic) treatment in miR-26a-3p-knockdown rats increased sucrose consumption in the sucrose preference test and (F) decreased immobility times and increased swimming times in the forced-swim test. (G) bpV(pic) treatment in DG neurons produced changes in spontaneous burst activity. $n=16$ rats per group. Each data point represents 1 animal. Electrophysiological recordings were repeated at least 3 times. Data are presented as mean \pm SEM. $n=18$ rats per group in behavioral tests. ${ }^{* *} P<0.01$, ${ }^{* *} P<0.001$ vs. WT; ${ }^{*} P<0.01$,

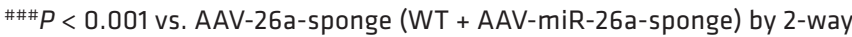
ANOVA with Tukey's post hoc correction.

20 micrographs were randomly selected from each rat for analysis using ImageJ software (NIH).

Reverse transcription PCR and quantitative real-time PCR. Total RNA from DG regions was isolated using the RNA rapid extraction kit (Aidlab) according to the manufacturer's instructions. Total RNA was reverse transcribed into cDNA and subsequently amplified by PCR with specific primers (Supplemental Table 1). PCR products were resolved by electrophoresis and images were obtained using the Gel Image Analysis System (Bio-Rad). Intensities of bands were analyzed using Image-Pro Plus 6.0 software and values were normalized to GAPDH.

Quantitative real-time PCR was performed with the Bio-Rad IQ5 Real-Time PCR System. The relative fold change in expression of miRNA was determined using the $2^{-\Delta \Delta C t}$ method. GAPDH served as a loading control in each group.

Western blot analysis. DG regions were carefully isolated and immediately homogenized in lysis buffer with a cocktail of protease inhibitors. Protein concentrations were determined using the BCA assay kit (Beyotime). Proteins (30 $\mu$ g) from each sample were electrophoretically resolved in 8\%-15\% SDS-PAGE gels, transferred to PVDF membranes, and probed with the following primary antibodies: anti-BDNF (1:300, catalog sc-546, Santa Cruz Biotechnology Inc.); anti-CREB (1:500, catalog 9197), anti-PSD-95 (1:1000, catalog 3450), anti-Syn (1:1000, catalog 5461), anti-LC3-I/LC3-II (1:1000, catalog 12741), anti-beclin-1 (1:1000, catalog 3495), anti-PARP (1:1000, catalog 9532), anti-cleaved caspase-3 (1:500, catalog 9661), anti-PTEN (1:500, catalog 9552), antiPI3K (1:500, catalog 4292), anti-p-Akt (1:500, catalog 9271), anti-p53 (1:500, catalog 9284), anti-p62 (1:1000, catalog 23214), anti- $\beta$-actin (1:1000, catalog 4970) (all Cell Signaling Technologies); anti-caspase-9 (1:500, catalog AP0359, Bioworld); and anti-BAX (1:1000, catalog 50599-2-lg) and anti-GAPDH (1:4000, catalog 10494-1-AP) (both Proteintech Group). The secondary antibodies were Peroxidase-conjugated goat anti-rabbit IgG (1:5000, catalog ZB-2301, Zhongshan Golden Bridge Biotechnology) and Peroxidase-conjugated goat anti-mouse (1:2000, catalog ZB-2305, Zhongshan Golden Bridge Biotechnology). Protein band densities were quantified using ImageJ software. The experiment with the samples of each rat was replicated at least three times and final data are expressed as a percentage of the control group.

Statistics. All statistical procedures were performed using GraphPad Prism 5. All data are presented as the mean \pm standard error of the mean (SEM). Pearson's coefficient tests were performed to analyze statistical significance in expression levels of miRNAs between normal controls and CUMS samples in the small-RNA sequencing analysis. The remaining data were analyzed with 1- or 2-way analysis of variance (ANOVA) followed by the Tukey's post hoc test for multiple comparisons of means. Student's $t$ tests were employed for comparisons between 2 groups if appropriate. A $P$ value of less than 0.05 was required for results to be considered statistically significant.

Study approval. All experimental procedures were approved by the Shandong University Animal Care and Use Committee (ECSBMSSDU-2018-2-056) and conform to the NIH Guide for the Care and Use of Laboratory Animals (National Academies Press, 2011).

\section{Author contributions}

SYY conceived of and designed the research study. YL acquired and analyzed data. YL, CF, LW, TL, RG, and WW performed the experiments. SYY wrote the manuscript.

\section{Acknowledgments}

This study was supported by grants to SYY from the National Natural Science Foundation of China (NSFC81873796 and NSFC82071513), the Natural Science Foundation of Shandong Province of China (ZR2020ZD25), and the Fundamental Research Funds of Shandong University (2018JC008). We thank the staff of the electron microscopy laboratory of the Morphological Experimental Center in the School of Basic Medical Sciences, Cheeloo College of Medicine, for technical assistance.

Address correspondence to: Shu Yan Yu, Wenhuaxilu Road 44\#, Jinan, Shandong Province, 250012, PR China. Phone: 86.0531.88383902; Email: shuyanyu@sdu.edu.cn.
1. Oh DH, et al. Neuropathological abnormalities of astrocytes, GABAergic neurons, and pyramidal neurons in the dorsolateral prefrontal cortices of patients with major depressive disorder. Eur Neuropsychopharmacol. 2012;22(5):330-338.

2. Stockmeier CA, et al. Cellular changes in the postmortem hippocampus in major depression. Biol Psychiatry. 2004;56(9):640-650.

3. Boldrini M, et al. Hippocampal granule neuron number and dentate gyrus volume in antidepressant-treated and untreated major depression. Neuropsychopharmacology. 2013;38(6):1068-1077.

4. Krishnan V, Nestler EJ. The molecular neurobiology of depression. Nature. 2008;455(7215):894-902.

5. Bastos AG, et al. The efficacy of long-term psychodynamic psychotherapy, fluoxetine and their combination in the outpatient treatment of depression. Psychother Res. 2015;25(5):612-624.

6. Gaynes BN, et al. What did $\mathrm{STAR}^{*} \mathrm{D}$ teach us? Results from a large-scale, practical, clinical trial for patients with depression. Psychiatr Serv. 2009;60(11):1439-1445.

7. Adzic M, et al. Therapeutic strategies for treatment of inflammation-related depression. Curr Neuropharmacol. 2018;16(2):176-209.

8. Wong $\mathrm{CH}$, et al. Estimation of clinical trial suc- 
cess rates and related parameters. Biostatistics. 2019;20(2):366-366

9. Savitz J, Drevets WC. Bipolar and major depressive disorder: Neuroimaging the develop mental-degenerative divide. Neurosci Biobehav Rev. 2009;33(5):699-771.

10. Goldwater DS, et al. Structural and functional alterations to rat medial prefrontal cortex following chronic restraint stress and recovery. Neuroscience. 2009;164(2):798-808.

11. Fernandes J, Gupta GL. N-acetylcysteine attenuates neuroinflammation associated depressive behavior induced by chronic unpredictable mild stress in rat. Behav Brain Res. 2019;364:356-365.

12. Park SC. Neurogenesis and antidepressant action. Cell Tissue Res. 2019;377(1):95-106

13. Kubera M, et al. In animal models, psychosocial stress-induced (neuro)inflammation, apoptosis and reduced neurogenesis are associated to the onset of depression. Prog Neuropsychopharmacol Biol Psychiatry. 2011;35(3):744-759.

14. Presutti C, et al. Non coding RNA and brain. $B M C$ Neurosci. 2006;7(suppl 1):S5

15. Pasquinelli AE. Non-coding RNA microRNAs and their targets: recognition, regulation and an emerging reciprocal relationship. Nat Rev Genet. 2012;13(4):271-282.

16. Nowak JS, Michlewski G. miRNAs in development and pathogenesis of the nervous system. Biochem Soc Trans. 2013;41(4):815-820.

17. Marangon D, et al. MicroRNAs change the games in central nervous system pharmacology. Biochem Pharmacol. 2019;168:162-172.

18. Su LN, et al. Network analysis of microRNAs, transcription factors, and target genes involved in axon regeneration. JZhejiang Univ Sci B. 2018;19(4):293-304.

19. McNeill E, Van Vactor D. MicroRNAs shape the neuronal landscape. Neuron. 2012;75(3):363-379.

20. Cao DD, et al. MicroRNAs: key regulators in the central nervous system and their implication in neurological diseases. Int J Mol Sci. 2016;17(6):842.

21. Lopez JP, et al. MicroRNAs $146 \mathrm{a} / \mathrm{b}-5$ and 425 $3 p$ and $24-3 p$ are markers of antidepressant response and regulate MAPK/Wnt-system genes. Nat Commun. 2017;8:15497.

22. Gibbons A, et al. Changes in non-coding RNA in depression and bipolar disorder: can they be used as diagnostic or theranostic biomarkers? Noncoding RNA. 2020;6(3):33.

23. Solich J, et al. Restraint stress in mice alters set of 25 miRNAs which regulate stress- and depression-related mRNAs. Int J Mol Sci. 2020;21(24):E9469.

24. Vlachos IS, et al. DIANA miRPath v.2.0: investigating the combinatorial effect of microRNAs in pathways. Nucleic Acids Res. 2012;40(W1):W498-W504.

25. Malhi GS, Mann JJ. Depression. Lancet. 2018;392(10161):2299-2312.

26. Willner P, et al. The neurobiology of depression and antidepressant action. Neurosci Biobehav Rev. 2013;37(10):2331-2371.

27. Fan CQ, et al. Curcumin protects against chronic stress-induced dysregulation of neuroplasticity and depression-like behaviors via suppress- ing IL-1 beta pathway in rats. Neuroscience. 2018;392:92-106.

28. Wang P, et al. Interleukin-6: its role and mechanisms in rescuing depression-like behaviors in rat models of depression. Brain Behav Immun. 2019;82:106-121.

29. Fan CQ, et al. Neuroprotective effects of ginsenoside-Rg1 against depression-like behaviors via suppressing glial activation, synaptic deficits, and neuronal apoptosis in rats. Front Immunol. 2018;9:2889.

30. Miao C, Chang J. The important roles of microRNAs in depression: new research progress and future prospects. J Mol Med (Berl). 2021;99(5):619-636.

31. Huang DW, et al. Systematic and integrative analysis of large gene lists using DAVID bioinformatics resources. Nat Protoc. 2009;4(1):44-57.

32. Kanehisa M, et al. From genomics to chemical genomics: new developments in KEGG. Nucleic Acids Res. 2006;34(suppl_1):D354-D357.

33. Xie L, et al. MicroRNA-26a-2 maintains stress resiliency and antidepressant efficacy by targeting the serotonergic autoreceptor HTR1A. Biochem Biophys Res Commun. 2019;511(2):440-446.

34. Huse JT, et al. The PTEN-regulating microRNA miR-26a is amplified in high-grade glioma and facilitates gliomagenesis in vivo. Genes Dev. 2009;23(11):1327-1337.

35. Mavrakis KJ, et al. A cooperative microRNA-tumor suppressor gene network in acute T-cell lymphoblastic leukemia (T-ALL). Nat Genet. 2011;43(7):673-678.

36. Liu B, et al. MiR-26a enhances metastasis potential of lung cancer cells via AKT pathway by targeting PTEN. Biochim Biophys Acta. 2012;1822(11):1692-1704

37. van Dijk MT, et al. Altered dentate gyrus microstructure in individuals at high familial risk for depression predicts future symptoms. Biol Psychiatry Cogn Neurosci Neuroimaging. 2021;6(1):50-58.

38. Santos MAO, et al. Global hippocampal atrophy in major depressive disorder: a meta-analysis of magnetic resonance imaging studies. Trends Psychiatry Psychother. 2018;40(4):369-378.

39. Roman-Albasini L, et al. Antidepressantrelevant behavioral and synaptic molecular effects of long-term fasudil treatment in chronically stressed male rats. Neurobiol Stress. 2020;13:100234.

40. Schratt GM, et al. A brain-specific microRNA regulates dendritic spine development. Nature. 2006;441(7095):902-902.

41. Jiang JJ, et al. MicroRNA-26a supports mammalian axon regeneration in vivo by suppressing GSK3 beta expression. Cell Death Dis. 2015;6(8):e1865.

42. Lucci C, et al. Spatiotemporal regulation of GSK3 beta levels by miRNA-26a controls axon development in cortical neurons. Development. 2020;147(3):dev180232

43. Li F, et al. miR-26a prevents neural stem cells from apoptosis via $\beta$-catenin signaling pathway in cardiac arrest-induced brain damage. Biosci Rep. 2019;39(5):BSR20181635.

44. Lafourcade CA, et al. A role for mir-26a in stress: a potential sEV biomarker and modulator of excitatory neurotransmission. Cells. 2020;9(6):1364.

45. Lai JP, et al. Phosphatase and tensin homologue deleted on chromosome ten (PTEN) as a molecular target in lung epithelial wound repair. Br JPharmacol. 2007;152(8):1172-1184.

46. Wang X, et al. Resveratrol protects the integrity of alveolar epithelial barrier via SIRT1/PTEN/p-Akt pathway in methamphetamine-induced chronic lung injury. Cell Prolif. 2020;53(3):e12773.

47. Qin Y, et al. mir-106a regulates cell proliferation and apoptosis of colon cancer cells through targeting the PTEN/PI3K/AKT signaling pathway. Oncol Lett. 2018;15(3):3197-3201.

48. Huang EJ, Reichardt LF. Neurotrophins: roles in neuronal development and function. Annu Rev Neurosci. 2001;24:677-736.

49. Leal G, et al. BDNF-induced local protein synthe sis and synaptic plasticity. Neuropharmacology. 2014;76:639-656.

50. Baehrecke EH. Autophagy: dual roles in life and death? Nat Rev Mol Cell Biol. 2005;6(6):505-510.

51. Ding J, et al. Inhibition of phosphatase and tensin homolog deleted on chromosome 10 decreases rat cortical neuron injury and blood-brain barrier permeability, and improves neurological functional recovery in traumatic brain injury model. PLoS One. 2013;8(11):e80429.

52. Borges GA, et al. Pharmacological PTEN inhibition: potential clinical applications and effects in tissue regeneration. Regen Med. 2020;15(2):1329-1344.

53. Pulido R. PTEN inhibition in human disease therapy. Molecules. 2018;23(2):E285.

54. Zhou J, et al. Activation of the PTEN/mTOR/ STAT3 pathway in breast cancer stem-like cells is required for viability and maintenance. Proc Natl Acad Sci U S A. 2007;104(41):16158-16163.

55. Parsa AT, et al. Loss of tumor suppressor PTEN function increases B7-H1 expression and immunoresistance in glioma. Nat Med. 2007;13(1):8488.

56. Martelli AM, et al. Targeting the translational apparatus to improve leukemia therapy: roles of the PI3K/PTEN/Akt/mTOR pathway. Leukemia 2011;25(7):1064-1079.

57. Mao QQ, et al. Peony glycosides produce antidepressant-like action in mice exposed to chronic unpredictable mild stress: effects on hypothalamic-pituitary-adrenal function and brainderived neurotrophic factor. Prog Neuropsychopharmacol Biol Psychiatry. 2009;33(7):1211-1216.

58. Porsolt RD, et al. Depression: a new animal model sensitive to antidepressant treatments. Nature. 1977;266(5604):730-732.

59. Duman CH, et al. A role for MAP kinase signaling in behavioral models of depression and antidepressant treatment. Biol Psychiatry. 2007;61(5):661-670.

60. Li Z, et al. MicroRNA-23b promotes avian leukosis virus subgroup J (ALV-J) replication by targeting IRF1. Sci Rep. 2015;5:10294.

61. Zhang QG, et al. Critical role of PTEN in the coupling between PI3K/Akt and JNK1/2 signaling in ischemic brain injury. FEBS Lett. 2007;581(3):495-505. 